\title{
Towards the complete analysis of the rotational spectrum of $\left(\mathrm{CH}_{3}\right)_{3} \mathrm{SnCl}$
}

\author{
Melanie Schnell \\ Universität Hannover, Institut für Physikalische Chemie, Lehrgebiet A, Callinstr. 3-3a, \\ D-30167 Hannover, Germany \\ Optical Technology Division, National Institute of Standards and Technology, \\ Gaithersburg, MD 20899, USA \\ Jon T. Hougen \\ Optical Technology Division, National Institute of Standards and Technology, \\ Gaithersburg, MD 20899, USA \\ Jens-Uwe Grabow \\ Universität Hannover, Institut für Physikalische Chemie, Lehrgebiet A, Callinstr. 3-3a, \\ D-30167 Hannover, Germany
}

\begin{abstract}
The rotational spectrum of the symmetric top trimethyl tin chloride $\left(\mathrm{CH}_{3}\right)_{3} \mathrm{SnCl}$ has been studied using a pulsed molecular beam Fourier transform microwave spectrometer in the frequency range from 3 to $24 \mathrm{GHz}$. The spectrum is exceedingly complicated by the internal rotation motions of the three equivalent methyl tops, the high number of $\mathrm{Sn}$ - and $\mathrm{Cl}$-isotopes and the quadrupole hyperfine structure of the chlorine nucleus. In this paper, we present the microwave spectrum, ab initio calculations, permutation inversion (PI) group-theoretical considerations, Starkeffect measurements and finally the assignments and fits of the different torsionrotation species. Based on the Stark-effect measurements, the dipole moment is $\mu=$ 3.4980(30) D. Due to $\Delta K= \pm 1$-mixing effects we observe linear Stark-effect behavior and additional quadrupole splitting for some $K=0$ torsion-rotation transitions in $\left(\mathrm{CH}_{3}\right)_{3} \mathrm{SnCl}$, which can be group-theoretically explained. The symmetric rotor fit of $A_{1}$ states leads to an effective $B$-constant of $1680.040124(50) \mathrm{MHz}$ for the main isotopologue $\left(\mathrm{CH}_{3}\right)_{3}{ }^{120} \mathrm{Sn}^{35} \mathrm{Cl}$. A global fit of $182 \mathrm{~K}=0$ torsion-rotation transitions yields a $V_{3}$ torsional barrier of $148.299(54) \mathrm{cm}^{-1}$.
\end{abstract}

Key words:

PACS: 


\section{Introduction}

The molecules of the type $\left(\mathrm{CH}_{3}\right)_{3} \mathrm{XY}$ considered here are symmetric tops with $\mathrm{C}_{3 v}$ symmetry in their equilibrium geometry. The rotational spectra of such molecules are especially interesting since they can show splittings arising from three chemically equivalent methyl tops, or, from a grouptheoretical point of view, splittings from three $C_{3 v}$-equivalent, individually $C_{3 v}$-symmetric rotors. From these splittings information on the torsional barrier height and on torsion-torsion interaction terms can be obtained. In the past only very few spectroscopic studies on molecules of the type $\left(\mathrm{CH}_{3}\right)_{3} \mathrm{XY}$ have been reported in which the torsion-rotation splitting is resolved $[1,2,3,4,5]$.

In general, both steric repulsion and properties of the chemical bonds are responsible for the barrier height to internal rotation, and their relative contributions in varying molecular situations is in general different. We recently presented a comparative study of the different origins of the barriers to internal rotation for the series $\left(\mathrm{CH}_{3}\right)_{3} \mathrm{XCl}$ with $\mathrm{X}=\mathrm{C}, \mathrm{Si}, \mathrm{Ge}, \mathrm{Sn}$ [4], since, although belonging to the same IVA main group in the periodic table, these four elements are quite different. The study aimed at a better understanding of the influence of the covalent radius and the properties of the central atom on the internal rotation barrier and on the top-top communication. Here, we present an in-depth analysis of the torsion-rotation spectrum of $\left(\mathrm{CH}_{3}\right)_{3} \mathrm{SnCl}$, since this molecule shows the most complex and thus most interesting spectral features.

For $\left(\mathrm{CH}_{3}\right)_{3} \mathrm{CCl}$ the barrier to internal rotation is high, and torsional splittings have not been resolved so far [6]. $\left(\mathrm{CH}_{3}\right)_{3} \mathrm{SiCl}$ has recently been studied by microwave spectroscopy [1]. The barrier to internal rotation was determined to be $V_{3}=577 \mathrm{~cm}^{-1}$, with observed torsional splitting pattern widths ranging from $50 \mathrm{kHz}$ for $J+1 \leftarrow J=1 \leftarrow 0$ to about $240 \mathrm{kHz}$ ( $5 \leftarrow 4$-transition). For $\left(\mathrm{CH}_{3}\right)_{3} \mathrm{GeCl}$ a rotational spectrum was recorded from $18 \mathrm{GHz}$ to $40 \mathrm{GHz}$ in an earlier investigation without resolving the torsional and the chlorine quadrupole hyperfine splittings [7]. We recently recorded and analysed the high-resolution microwave spectrum of $\left(\mathrm{CH}_{3}\right)_{3} \mathrm{GeCl}$ including hyperfine structure and torsional splittings [3]. The barrier height is determined to be $V_{3}=372.359(47) \mathrm{cm}^{-1}\left(\left(\mathrm{CH}_{3}\right)_{3}{ }^{74} \mathrm{Ge}{ }^{35} \mathrm{Cl}\right)$. A detailed investigation of the torsion-rotation splitting pattern of $\left(\mathrm{CH}_{3}\right)_{3} \mathrm{SnCl}$ might lead to a better understanding of internal rotation dynamics since the torsional barrier can be expected to be mainly determined by properties of the three Sn-C bonds, rather than by steric effects, as briefly discussed in [4]. Following this reasoning, the barrier to internal rotation should be relatively low, leading to

Email address: schnell@fhi-berlin.mpg.de (Melanie Schnell). 
large splittings in the microwave spectrum.

The aim of the present study is to investigate the torsion-rotation spectrum of $\left(\mathrm{CH}_{3}\right)_{3} \mathrm{SnCl}$ to reveal the competing mechanisms of the internal dynamics. The remaining parts of the paper are divided as follows: In section 2 we describe the experimental details while in section 3 the observed rotational spectrum is discussed from a more qualitative point of view. In section 4 the group-theoretical aspects are described, section 5 continues with the analysis of Stark-effect measurements in order to differentiate between different torsional species and to determine the dipole moment of $\left(\mathrm{CH}_{3}\right)_{3} \mathrm{SnCl}$. In section 6 the results of the spectroscopic investigation are discussed. Section 7 concludes the paper and gives an outlook.

\section{Experimental}

$\left(\mathrm{CH}_{3}\right)_{3} \mathrm{SnCl}$, with a stated purity of $97 \%$, was purchased from Sigma-Aldrich and was used without further purification. $\left(\mathrm{CH}_{3}\right)_{3} \mathrm{SnCl}$ is a solid at room temperature and melts between $37-39^{\circ} \mathrm{C}$. Its vapor pressure is sufficiently high to prepare mixtures of about $1 \%$ substance in neon at stagnation pressures between $50-100 \mathrm{kPa}$, and thus to record its rotational spectrum. A broadband $(2-26.5 \mathrm{GHz})$ high resolution Fourier transform microwave (FTMW) spectrometer, shown in Figure 1, was used to observe the rotational spectrum of $\left(\mathrm{CH}_{3}\right)_{3} \mathrm{SnCl}$. The coaxially oriented beam resonator arrangement (COBRA) [8] is utilized, resulting in line widths of about $1 \mathrm{kHz}$ (half width at half height (HWHH)) for argon and about $1.5 \mathrm{kHz}(\mathrm{HWHH})$ for neon as carrier gas. This yields a resolving power of about $3 \mathrm{kHz}$ for argon and about $5 \mathrm{kHz}$ for neon, respectively. The spectrometer was designed to provide high sensitivity and resolution also in the low frequency range below $6 \mathrm{GHz}$, which was particularly helpful for this study since the $J+1 \leftarrow J=1 \leftarrow 0$-transition of $\left(\mathrm{CH}_{3}\right)_{3} \mathrm{SnCl}$ lies near $3.2 \mathrm{GHz}$.

To differentiate between different torsional species by their Stark behavior and to precisely determine the dipole moment of $\left(\mathrm{CH}_{3}\right)_{3} \mathrm{SnCl}$ we performed Stark-effect measurements using the CAESAR setup (coaxially aligned electrodes for Stark-effect arranged in resonators) [9] where the reflectors forming the microwave resonator are used as Stark electrodes themselves, supplemented by additional ring electrodes. In this arrangement, which is illustrated in Figure 1, the advantage of the longer transit time of the molecular jet in the coaxial arrangement is particularly useful for Stark-effect experiments, where very narrow line widths are desired. This is especially true for $\left(\mathrm{CH}_{3}\right)_{3} \mathrm{SnCl}$, which exhibits a very dense line pattern in the rotational spectrum. In this setup the direction of the static electric field is perpendicular to the electromagnetic field, leading exclusively to the selection rules 


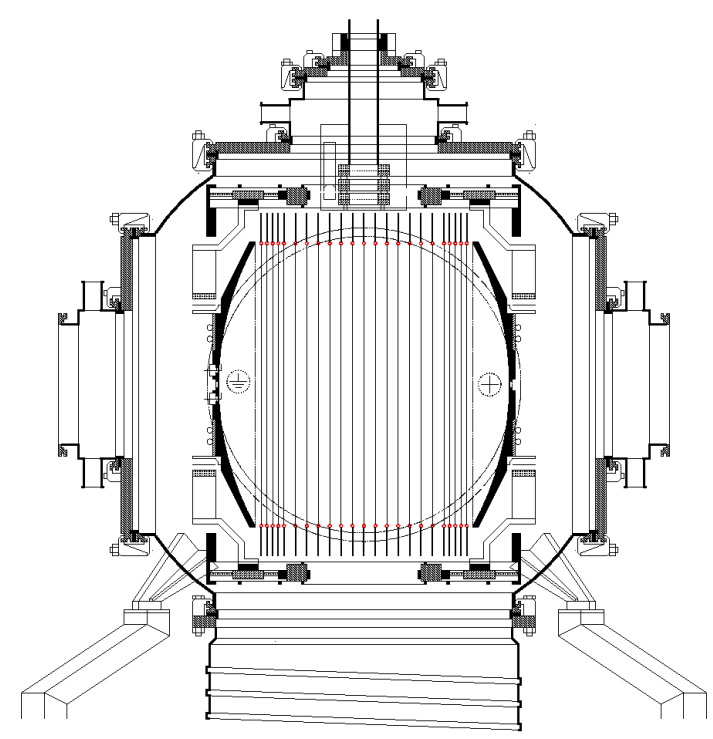

Figure 1. Experimental setup of the FTMW spectrometer including an assembly for Stark-effect measurements. The molecules are supersonically expanded into the resonator through a pulsed nozzle located in the left reflector (COBRA arrangement [8]). The microwave antennae are mounted on the same reflector. For Stark-effect measurements the right reflector is raised to a high voltage with respect to the left reflector, which is kept at ground potential. Additional ring electrodes between the two reflectors insure a homogeneous electric field along the resonator axis (CAESAR electrode arrangement [9]).

$\Delta M_{J}= \pm 1$. The Stark electric field $E$ was determined from calibration measurements with the $\mathrm{OC}^{36} \mathrm{~S}$ and ${ }^{18} \mathrm{OCS}$ isotopologues using a dipole moment of $\mu=0.71519$ (3) D for both species [10].

\section{Geometry considerations and spectral observations}

Our ab initio calculations show that $\left(\mathrm{CH}_{3}\right)_{3} \mathrm{SnCl}$ exhibits $\mathrm{C}_{3 v}$-symmetry in its equilibrium configuration, both for its $\mathrm{SnC}_{3} \mathrm{Cl}$ frame, as well as for the orientations of the three $\mathrm{CH}_{3}$ groups. Figure 2 displays the structure obtained from our BP86/def-TZVPP/ecp-46-mwb(Sn) optimization with no predetermined symmetry imposed. As a result of this high symmetry, the rotational spectrum should follow that for a prolate symmetric top. The predicted rotational constants are $A=2330.67 \mathrm{MHz}$ and $B=C=1614.21 \mathrm{MHz}$.

The observed rotational spectrum is complicated by rather dense line patterns due to internal rotation of the three methyl tops, chlorine nuclear quadrupole hyperfine structure, and the large number of isotopes of tin and chlorine. Based on the $a b$ initio calculations (BP86/def-TZVPP/ecp-46$\mathrm{mwb}(\mathrm{Sn}))$ we estimate a torsional barrier of about $2.1 \mathrm{~kJ} / \mathrm{mol}\left(175 \mathrm{~cm}^{-1}\right)$ for one methyl group. 


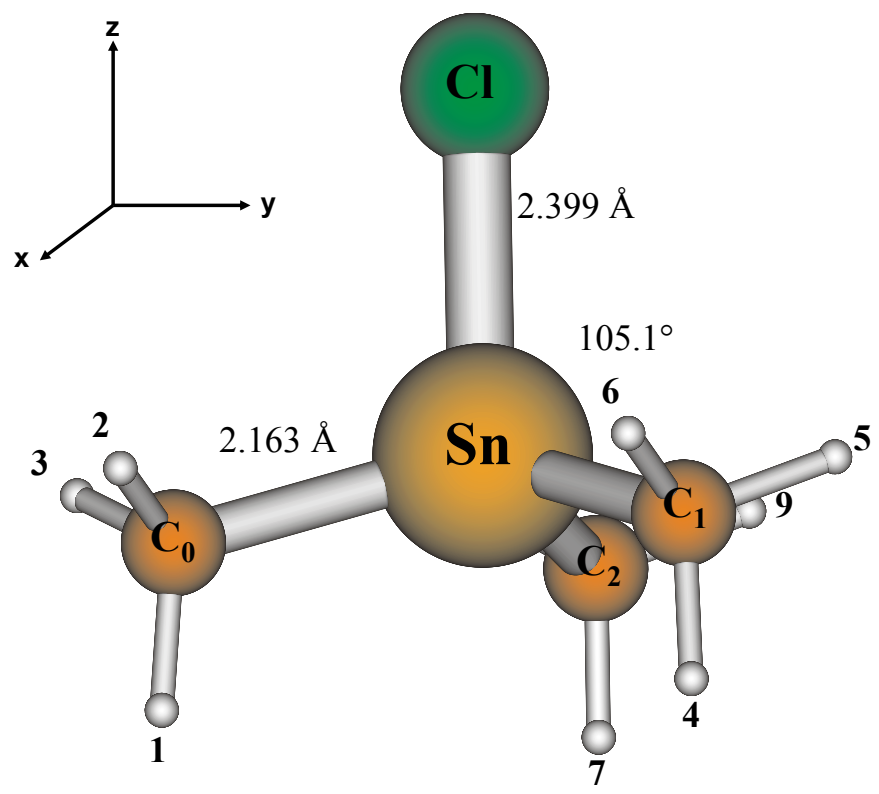

Figure 2. Equilibrium geometry (BP86/def-TZVPP/ecp-46-mwb(Sn)) and numbering of the identical nuclei of $\left(\mathrm{CH}_{3}\right)_{3} \mathrm{SnCl}$. This configuration corresponds to framework 1 in the high-barrier torsion-rotation formalism described in section 4.4.

Figure 3 shows a scheme of the different splitting patterns and subgroups observed for $\left(\mathrm{CH}_{3}\right)_{3} \mathrm{SnCl}(J+1 \leftarrow J=5 \leftarrow 4$-transition). The $5 \leftarrow 4$-transition is spread over a spectral range of about $1.2 \mathrm{GHz}$. It exhibits two main groups of about $400 \mathrm{MHz}$ width corresponding to the ${ }^{35} \mathrm{Cl}-$ and ${ }^{37} \mathrm{Cl}$ isotopologues, separated by about $400 \mathrm{MHz}$. Both groups are further split into 17 groups due to internal rotation, six for $K=0$ and eleven for $K=1$, which have a maximum separation of about $50 \mathrm{MHz}$. Each torsional group is now further separated into seven more groups corresponding to the seven $\mathrm{Sn}$ isotopologues $\left({ }^{124} \mathrm{Sn}\left(5.98 \%\right.\right.$ natural relative abundance), ${ }^{122} \mathrm{Sn}(4.71 \%),{ }^{120} \mathrm{Sn}$ $\left.(32.97 \%),{ }^{119} \mathrm{Sn}(8.58 \%),{ }^{118} \mathrm{Sn}(24.01 \%),{ }^{117} \mathrm{Sn}(7.57 \%),{ }^{116} \mathrm{Sn}(14.24 \%)\right)$, which are separated by about $10 \mathrm{MHz}$ and which have been detected in natural abundance. The narrowest pattern we observed is due to quadrupole coupling of the chlorine nucleus $\left(I=\frac{3}{2}\right)$. In total, one single $J+1 \leftarrow J$-symmetric top rotational transition with $J>0$ shows more than 1000 lines in the spectrum.

Figure 4a shows the measured rotation spectrum between $16507 \mathrm{MHz}$ and $16917 \mathrm{MHz}$, which corresponds to the $J+1 \leftarrow J=5 \leftarrow 4$-transition of the $\left(\mathrm{CH}_{3}\right)_{3} \mathrm{Sn}^{35} \mathrm{Cl}$ isotopologue (part a). The braces indicate different torsionrotation states for $K=1$ and $K=0$, denoted by roman and arabic numbering, respectively. Up to now we were able to unambiguously identify 14 torsional states (six for $K=0$ and eight for $K=1$ ) with the help of the very characteristic tin isotopolog pattern, while we expect the missing three $K=1$-torsional states to be located in the very dense line pattern, where also the torsional 


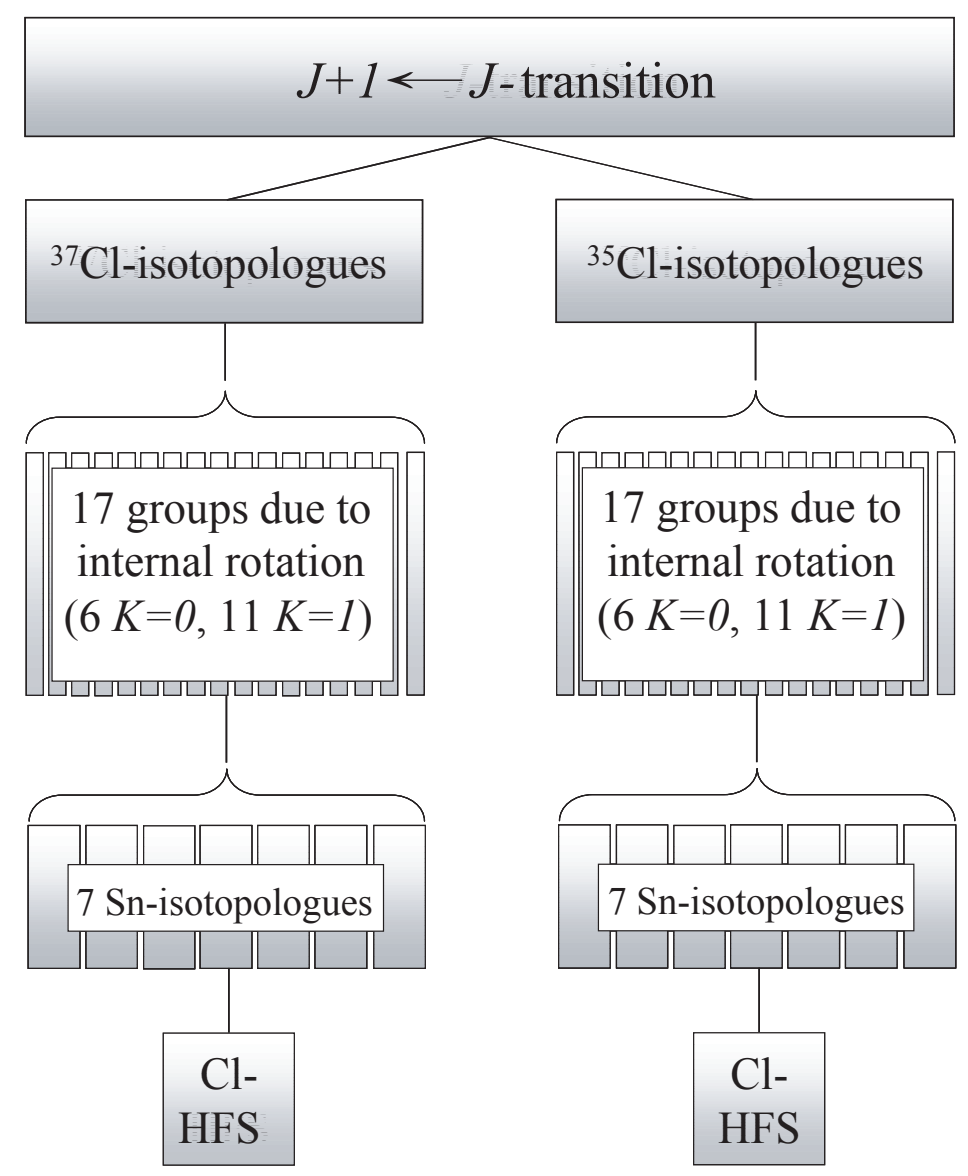

Figure 3. Schematic representation of the characteristics of the rotational spectrum of $\left(\mathrm{CH}_{3}\right)_{3} \mathrm{SnCl}$. One rotational $J+1 \leftarrow J$-transition is divided into several subgroups because of $\mathrm{Cl}$ - and $\mathrm{Sn}$ isotopologues, internal rotation of the three equivalent $\mathrm{CH}_{3}$ groups and $\mathrm{Cl}$ nuclear quadrupole hyperfine structure. The $J+1 \leftarrow J=5 \leftarrow 4$-transition spreads over $1.2 \mathrm{GHz}$. It is further divided into two subgroups, one for the ${ }^{37} \mathrm{Cl}$ - and one for the ${ }^{35} \mathrm{Cl}$-isotopologue. These two groups are each spread over about $400 \mathrm{MHz}$ and are separated by about $400 \mathrm{MHz}$. They are further split due to internal rotation, the seven tin isotopologues and chlorine nuclear quadrupole coupling, which is the smallest splitting observed (0.1-2 MHz).

groups VIII, IX, 10, and 11 are found. The tin isotopolog pattern displayed in Figure $4 \mathrm{~b}$ (labeled with their mass numbers) facilitated first assignments of this very complex spectrum since the relative abundances of the different isotopologues are very nicely reproduced by the intensities in the spectrum. In Figure $4 \mathrm{c}$ the chlorine nuclear quadrupole hyperfine structure from the most intense quadrupole coupling components $F^{\prime} \leftarrow F=\frac{13}{2} \leftarrow \frac{11}{2}, \frac{11}{2} \leftarrow \frac{9}{2}$, $\frac{9}{2} \leftarrow \frac{7}{2}$, and $\frac{7}{2} \leftarrow \frac{5}{2}$ for $K=0$ is shown. Note, that the quadrupole coupling components $\frac{13}{2} \leftarrow \frac{11}{2}$ and $\frac{11}{2} \leftarrow \frac{9}{2}$ as well as $\frac{9}{2} \leftarrow \frac{7}{2}$ and $\frac{7}{2} \leftarrow \frac{5}{2}(K=0)$ are 


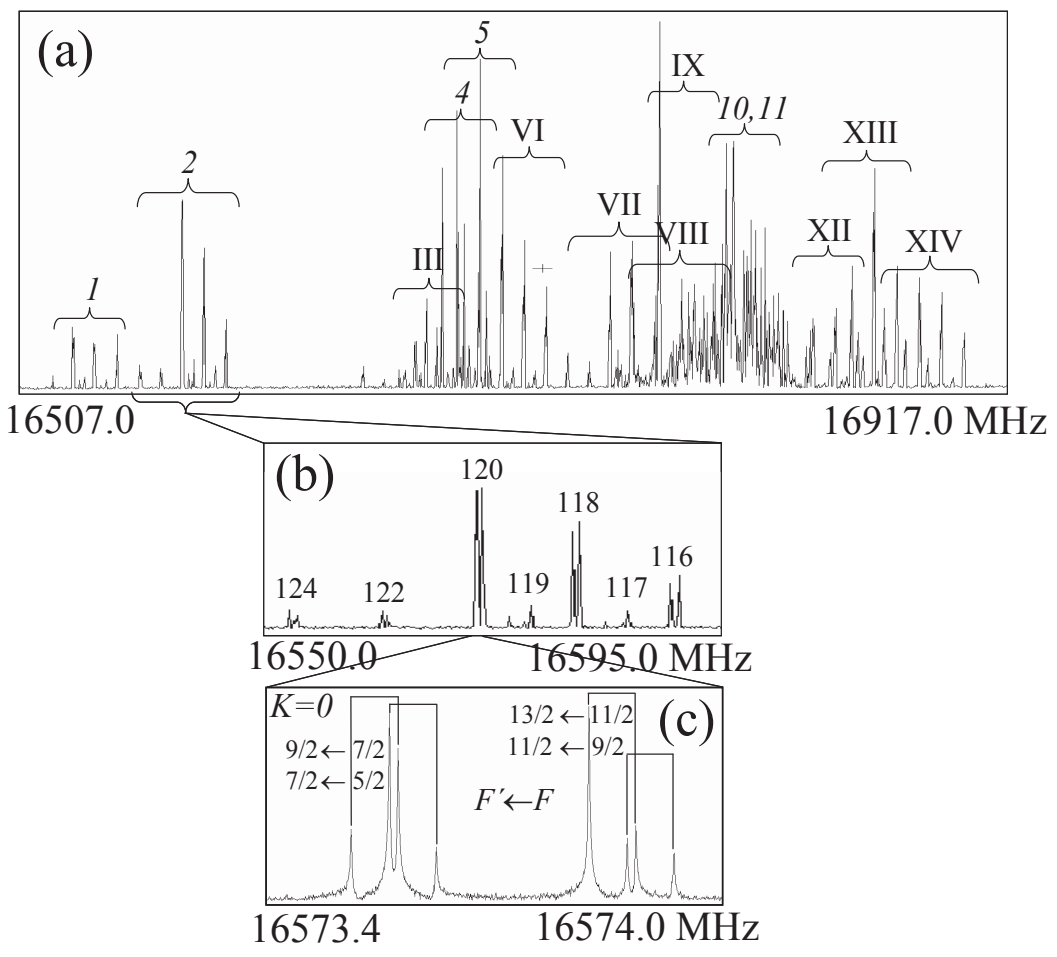

Figure 4. Parts of the rotational spectrum of $\left(\mathrm{CH}_{3}\right)_{3} \mathrm{SnCl}$. (a) the $J+1 \leftarrow J=5 \leftarrow 4$-transition of the main isotopologue $\left(\mathrm{CH}_{3}\right)_{3} \mathrm{Sn}^{35} \mathrm{Cl}$ between 16507 and $16917 \mathrm{MHz} .14$ groups due to internal rotation are identified (Roman numbers indicate $K=1$ and Arabic numbers $K=0$ torsion-rotation transitions). (b) seven different tin isotopologues, labeled by their $\mathrm{Sn}$ masses, with the characteristic intensity pattern reflecting the natural abundances of the different $\mathrm{Sn}$-isotopes, and (c) chlorine nuclear quadrupole hyperfine structure for $K=0$ labeled with the corresponding $F^{\prime} \leftarrow F$ quantum numbers of the transition.

usually unresolvably close in frequency for $\left(\mathrm{CH}_{3}\right)_{3} \mathrm{XCl}$ type molecules [11]. This is indeed observed for $\left(\mathrm{CH}_{3}\right)_{3} \mathrm{SiCl}$ [1] and $\left(\mathrm{CH}_{3}\right)_{3} \mathrm{GeCl}$ [3]. However, for $\left(\mathrm{CH}_{3}\right)_{3} \mathrm{SnCl}$ we find small differences in the transition frequency ranging from $40 \mathrm{kHz}$ to $450 \mathrm{kHz}$ for these components. These frequency differences are dependent on $J$ and on the torsional species; they will be discussed in more detail in section 6.2.

\section{Group Theory / Theoretical Considerations}

\subsection{The group $G_{162}$}

To facilitate the assignment and the fitting of spectroscopic constants to this complex rotational spectrum we used permutation-inversion (PI) group 
Table 1

Character Table of $\mathrm{G}_{162}$ with $\epsilon=\exp (2 \pi i / 3) . F_{4 a}$ and $F_{4 b}$ as well as $F_{5 a}$ and $F_{5 b}$ are separable degenerate to each other and can be combined to form the reducible representations $I_{4}$ and $I_{5}$ as given in the last two rows.

\begin{tabular}{lcccccccccccccc}
\hline \hline & $\mathrm{E}$ & $\alpha \beta \gamma$ & $\alpha \beta^{-1}$ & $\alpha^{-1} \beta$ & $\alpha$ & $\alpha \beta$ & $\alpha^{-1} \beta \gamma$ & $\mathrm{c}$ & $\alpha \mathrm{c}$ & $\alpha^{-1} \mathrm{c}$ & $\sigma$ & $\beta \sigma$ & $\beta^{-1} \sigma$ & \\
& 1 & 2 & 3 & 3 & 6 & 6 & 6 & 18 & 18 & 18 & 27 & 27 & 27 & \\
\hline $\mathrm{A}_{1}$ & 1 & 1 & 1 & 1 & 1 & 1 & 1 & 1 & 1 & 1 & 1 & 1 & 1 & $\mathrm{~T}_{z}$ \\
$\mathrm{~A}_{2}$ & 1 & 1 & 1 & 1 & 1 & 1 & 1 & 1 & 1 & 1 & -1 & -1 & -1 & $\mathrm{R}_{z}$ \\
$\mathrm{E}_{1}$ & 2 & 2 & 2 & 2 & 2 & 2 & 2 & -1 & -1 & -1 & 0 & 0 & 0 & $\left(\mathrm{~T}_{x}, \mathrm{~T}_{y}\right)\left(\mathrm{R}_{x}, \mathrm{R}_{y}\right)$ \\
$\mathrm{E}_{2}$ & 2 & 2 & 2 & 2 & -1 & -1 & -1 & 2 & -1 & -1 & 0 & 0 & 0 & \\
$\mathrm{E}_{3}$ & 2 & 2 & 2 & 2 & -1 & -1 & -1 & -1 & 2 & -1 & 0 & 0 & 0 & \\
$\mathrm{E}_{4}$ & 2 & 2 & 2 & 2 & -1 & -1 & -1 & -1 & -1 & 2 & 0 & 0 & 0 & \\
$\mathrm{I}_{1}$ & 6 & -3 & 0 & 0 & 0 & -3 & 3 & 0 & 0 & 0 & 0 & 0 & 0 & \\
$\mathrm{I}_{2}$ & 6 & -3 & 0 & 0 & 3 & 0 & -3 & 0 & 0 & 0 & 0 & 0 & 0 & \\
$\mathrm{I}_{3}$ & 6 & -3 & 0 & 0 & -3 & 3 & 0 & 0 & 0 & 0 & 0 & 0 & 0 & \\
$\mathrm{~F}_{4 a}$ & 3 & 3 & $3 \epsilon^{*}$ & $3 \epsilon$ & 0 & 0 & 0 & 0 & 0 & 0 & 1 & $\epsilon$ & $\epsilon^{*}$ & \\
$\mathrm{~F}_{4 b}$ & 3 & 3 & $3 \epsilon$ & $3 \epsilon^{*}$ & 0 & 0 & 0 & 0 & 0 & 0 & 1 & $\epsilon^{*}$ & $\epsilon$ & \\
$\mathrm{F}_{5 a}$ & 3 & 3 & $3 \epsilon^{*}$ & $3 \epsilon$ & 0 & 0 & 0 & 0 & 0 & 0 & -1 & $-\epsilon$ & $-\epsilon^{*}$ & \\
$\mathrm{~F}_{5 b}$ & 3 & 3 & $3 \epsilon$ & $3 \epsilon^{*}$ & 0 & 0 & 0 & 0 & 0 & 0 & -1 & $-\epsilon^{*}$ & $-\epsilon$ & \\
$\mathrm{I}_{4}$ & 6 & 6 & -3 & -3 & 0 & 0 & 0 & 0 & 0 & 0 & 2 & -1 & -1 & \\
$\mathrm{I}_{5}$ & 6 & 6 & -3 & -3 & 0 & 0 & 0 & 0 & 0 & 0 & -2 & 1 & 1 & \\
\hline \hline
\end{tabular}

theory. The PI group $\mathrm{G}_{162}$, appropriate for $\left(\mathrm{CH}_{3}\right)_{3} \mathrm{SnCl}$, as well as statistical weights for the torsional species have already been worked out $[12,13,14]$. $\mathrm{G}_{162}$ (Table 1) is formed from a direct product of three $\mathrm{C}_{3}$ groups $\left[\mathrm{C}_{3}\left(\mathrm{C}_{0}\right)\right.$ $\otimes C_{3}\left(C_{1}\right) \otimes C_{3}\left(C_{2}\right)=G_{27}$ for the three methyl groups following the numbering in Figure 2, respectively, and a semi-direct product with the $\mathrm{C}_{3 v}$ group (six elements) for the equilibrium symmetry [14]. It consists of 13 classes. With the numbering of the hydrogen and carbon nuclei given in Figure 2 the generating operations are $\alpha=(123), \beta=(456), \gamma=(789)$, $c=\left(C_{0}, C_{1}, C_{2}\right)(1,4,7)(2,5,8)(3,6,9)$ and $\sigma=\left(C_{1}, C_{2}\right)(2,3)(4,7)(5,9)(6,8)^{*}$. Due to the high symmetry of $\left(\mathrm{CH}_{3}\right)_{3} \mathrm{SnCl}$ the group $\mathrm{G}_{162}$ has four doubly degenerate species $\left(E_{i}, i=1-4\right)$, three sixfold-degenerate $\left(I_{j}, j=1-3\right)$, and four threefold-degenerate irreducible representations $\left(F_{4 a}, F_{4 b}\right.$ and $F_{5 a}$, $\left.F_{5 b}\right) . F_{4 a}, F_{4 b}$ and $F_{5 a}, F_{5 b}$, respectively, are separably degenerate $[15,16]$ because of time inversion and can be combined in pairs to give the reducible representations $I_{4}$ and $I_{5}$ [13], respectively, as shown in the last two rows of Table 1.

\subsection{The coordinate system}

The question of how a given symmetric rotor energy level of $\left(\mathrm{CH}_{3}\right)_{3} \mathrm{SnCl}$ will split when the three $\mathrm{CH}_{3}$ internal rotations become feasible can only 
be answered after the transformation properties of the rotational and other basis functions have been determined. These transformation properties can in turn only be determined after the relation between the laboratory-fixed and molecule-fixed coordinates has been defined. We follow closely the procedure described in Section II of Ref. [17], resulting in the following equation

$$
\mathbf{R}_{i}=\mathbf{R}+S^{-1}(\chi, \theta, \phi) \mathbf{a}_{i}\left(\alpha_{0}, \alpha_{1}, \alpha_{2}\right),
$$

which should be read from right to left. This procedure, in its simplest form, consists of choosing a set of constants $\mathbf{a}_{i}^{0}$, which describe a set of initial vector positions for the atoms $i$ in the molecule-fixed axis system. These initial positions, which are usually chosen as symmetrically as possible, are then subjected to the large amplitude motions, which for $\left(\mathrm{CH}_{3}\right)_{3} \mathrm{SnCl}$ consist of three internal rotation angles $\alpha_{0}, \alpha_{1}$, and $\alpha_{2}$, to generate a molecule of some particular instantaneous shape $\mathbf{a}_{i}^{\prime}\left(\alpha_{0}, \alpha_{1}, \alpha_{2}\right)$. These shape coordinates are then translated through a distance $\boldsymbol{A}$ to give coordinates $\mathbf{a}_{i}\left(\alpha_{0}, \alpha_{1}, \alpha_{2}\right)=$ $\mathbf{a}^{\prime}{ }_{i}\left(\alpha_{0}, \alpha_{1}, \alpha_{2}\right)-\mathbf{A}\left(\alpha_{0}, \alpha_{1}, \alpha_{2}\right)$, for which the origin coincides with the center of mass of the molecule. These center-of-mass coordinates are then substituted into Eq. 1, where they are rotated (using $S^{-1}(\chi, \theta, \phi)$ ) and translated (by $\mathbf{R}$ ) to give the proper molecular orientation and location in the laboratory-fixed axis system.

The positions $\mathbf{a}_{S n}$ and $\mathbf{a}_{C l}$ are simple to describe, since they are initially chosen to lie at the origin and on the $\mathrm{z}$ axis of the molecule, respectively, and (apart from center-of-mass effects) they do not vary with the internal rotation angles (e.g., $\mathbf{a}^{\prime}{ }_{S n}=\mathbf{a}_{S n}^{0}$ ):

$\mathbf{a}_{S n}\left(\alpha_{0}, \alpha_{1}, \alpha_{2}\right)=\mathbf{a}_{S n}^{\prime}-\mathbf{A}\left(\alpha_{0}, \alpha_{1}, \alpha_{2}\right)=\mathbf{a}_{S n}^{0}-\mathbf{A}\left(\alpha_{0}, \alpha_{1}, \alpha_{2}\right)=\mathbf{0}-\mathbf{A}\left(\alpha_{0}, \alpha_{1}, \alpha_{2}\right)$

$\mathbf{a}_{C l}\left(\alpha_{0}, \alpha_{1}, \alpha_{2}\right)=\mathbf{a}_{C l}^{\prime}-\mathbf{A}\left(\alpha_{0}, \alpha_{1}, \alpha_{2}\right)=\mathbf{a}_{C l}^{0}-\mathbf{A}\left(\alpha_{0}, \alpha_{1}, \alpha_{2}\right)=\left(\begin{array}{c}0 \\ 0 \\ r_{S n-C l}\end{array}\right)-\mathbf{A}\left(\alpha_{0}, \alpha_{1}, \alpha_{2}\right)$.

The positions of the atoms in each of three methyl groups are less straightforward to set up. Each of the three methyl tops consists of a carbon atom $\mathrm{C}_{j}(j=0,1,2)$ as anchor atom which is connected to the central tin atom and the hydrogen atoms $\mathrm{H}_{j k}(j=0,1,2 ; k=0,1,2)$, with, for example, $\mathrm{H}_{00}$ being $\mathrm{H}_{1}, \mathrm{H}_{10}$ being $\mathrm{H}_{4}$, and $\mathrm{H}_{22}$ being $\mathrm{H}_{9}$ in Figure 2 (framework 1 ), respectively. To describe the positions of the internal rotors and the internal rotation motions we first define the initial positions of all the carbon atoms as lying at 
the same point on the positive $\mathrm{Sn}-\mathrm{Cl}$ axis:

$$
\mathbf{a}_{C_{j}}^{0}=\left(\begin{array}{c}
0 \\
0 \\
r_{S n-C}
\end{array}\right),
$$

which will then be rotated about the $y$ axis by the Cl-Sn-C bond angle $\beta_{C}$ and, for $j=1$ and 2, out of the zx-plane to give

$$
\mathbf{a}_{C_{j}}=S^{-1}\left(0, \beta_{C}, 2 \pi j / 3\right) \mathbf{a}_{C}^{0}-A, j=0,1,2 .
$$

We assume $\beta_{C}=105.1^{\circ}$, as obtained from our ab initio optimization (Figure 2 ), to be constant. The positions of the hydrogen atoms $\mathrm{H}_{j k}(j=0,1,2 ; k=0,1,2)$ are obtained by connecting them to the corresponding $\mathrm{C}_{j}$-atom with the bond distance $r_{\mathrm{C}-\mathrm{H}}$, using a vector

$$
\mathbf{a}_{H}^{0}=\left(\begin{array}{c}
0 \\
0 \\
r_{C-H}
\end{array}\right) .
$$

This vector is first rotated about the $y$ axis by the complement $\pi-\beta_{H}$ of the Sn-C-H bond angle $\beta_{H}$, then rotated about the $z$ axis by an angle of $2 \pi \mathrm{k} / 3$ for each $\mathrm{H}_{j k}$, and finally attached to the $\mathrm{C}_{j}$ atom on the $\mathrm{z}$ axis to give

$$
\mathbf{a}_{H j k}=S^{-1}\left(0, \beta_{C}, 2 \pi j / 3\right)\left[\mathbf{a}_{C}^{0}+S^{-1}\left(0, \pi-\beta_{H}, \alpha_{j}+2 \pi k / 3\right) \mathbf{a}_{H}^{0}\right]-A,
$$

with $\beta_{H}$ taken to be constant at $109.5^{\circ}$. Eq. 7 indicates that a change of the torsional angle $\alpha_{j}$ leads only to a change of the positions of the hydrogen atoms connected to the carbon $C_{j}$. In general, we assume the skeletal bond distances and angles to be constant during the internal rotation motions, with the values for the configuration (framework 1) displayed in Figure 2.

We have now defined nine different variables to describe $\left(\mathrm{CH}_{3}\right)_{3} \mathrm{SnCl}$ : The three torsional angles $\alpha_{j}$, the three Eulerian angles $\chi, \theta$, and $\phi$ to transform from the molecule-fixed to the laboratory-fixed coordinate system, and the three coordinates for the molecular center-of-mass $\mathbf{R}$. The transformation properties of these nine variables under the generating symmetry operations $\alpha=(123), \beta=(456), \gamma=(789), c=\left(C_{0}, C_{1}, C_{2}\right)(1,4,7)(2,5,8)(3,6,9)$ and $\sigma=\left(C_{1}, C_{2}\right)(2,3)(4,7)(5,9)(6,8)^{*}$ of $G_{162}$, which are needed for the following considerations, are given in Table 2.

Based on these transformation properties the determination of the symmetry species of the different rotational and torsional states becomes possible. The torsional states and their symmetry species are given in Table 3, together 
Table 2

Transformation properties of the nine variables under the generating operations of $\mathrm{G}_{162}$.

\begin{tabular}{lccccc}
\hline & $\mathbf{R}$ & $\alpha_{0}$ & $\alpha_{1}$ & $\alpha_{2}$ & rotation \\
\hline$(123)$ & $\mathbf{R}$ & $\alpha_{0}+2 \pi / 3$ & $\alpha_{1}$ & $\alpha_{2}$ & $\chi, \theta, \phi$ \\
$(456)$ & $\mathbf{R}$ & $\alpha_{0}$ & $\alpha_{1}+2 \pi / 3$ & $\alpha_{2}$ & $\chi, \theta, \phi$ \\
$(789)$ & $\mathbf{R}$ & $\alpha_{0}$ & $\alpha_{1}$ & $\alpha_{2}+2 \pi / 3$ & $\chi, \theta, \phi$ \\
$c$ & $\mathbf{R}$ & $\alpha_{1}$ & $\alpha_{2}$ & $\alpha_{0}$ & $\chi+2 \pi / 3, \theta, \phi$ \\
$\sigma$ & $-\mathbf{R}$ & $-\alpha_{0}$ & $-\alpha_{2}$ & $-\alpha_{1}$ & $\pi-\chi, \pi-\theta, \pi+\phi$ \\
\hline \hline
\end{tabular}

Table 3

Symmetry species of the torsional tunneling levels and torsion-rotation energy levels [13] which arise when all three methyl rotors have only the zero-point torsional excitation, the corresponding nuclear spin weights for the torsion-rotation levels ${ }^{a}$, and their linear or quadratic Stark-effect behavior for $K=0$, as predicted from group theory and as experimentally determined from the corresponding torsion-rotation transitions.

\begin{tabular}{llllllll}
\hline \hline$\left|M_{a} M_{b} M_{c}\right\rangle^{b}$ & $\Gamma_{\text {torsional }}$ & \multicolumn{2}{c}{$K=0$} & $K=3 n \pm 1$ & $K=3 n$ & \multicolumn{2}{c}{$\begin{array}{l}\text { Stark-effect behavior } \\
\text { group theory }\end{array}$} \\
& & $J_{\text {even }}$ & $J_{\text {odd }}$ & & & exp. \\
\hline$|0,0,0\rangle$ & $\mathrm{A}_{1}$ & $\mathrm{~A}_{1}$ & $\mathrm{~A}_{2}$ & $\mathrm{E}_{1}$ & $\mathrm{~A}_{1}+\mathrm{A}_{2}$ & quad & quad \\
& & $(24)$ & $(24)$ & $(40)$ & $(24+24)$ & & \\
$| \pm 1,0,0\rangle$ & $\mathrm{I}_{2}$ & $\mathrm{I}_{2}$ & $\mathrm{I}_{2}$ & $2 \mathrm{I}_{2}$ & $2 \mathrm{I}_{2}$ & linear & linear \\
& & $(64)$ & $(64)$ & $(128)$ & $(128)$ & & \\
$| \pm 1, \pm 1,0\rangle$ & $\mathrm{I}_{1}$ & $\mathrm{I}_{1}$ & $\mathrm{I}_{1}$ & $2 \mathrm{I}_{1}$ & $2 \mathrm{I}_{1}$ & linear & linear \\
& & $(32)$ & $(32)$ & $(64)$ & $(64)$ & & \\
$| \pm 1, \mp 1,0\rangle$ & $\mathrm{I}_{4}$ & $\mathrm{I}_{4}$ & $\mathrm{I}_{5}$ & $\mathrm{I}_{4}+\mathrm{I}_{5}$ & $\mathrm{I}_{4}+\mathrm{I}_{5}$ & quad & quad \\
& & $(32)$ & $(32)$ & $(32+32)$ & $(32+32)$ & & \\
$| \pm 1, \pm 1, \mp 1\rangle$ & $\mathrm{I}_{3}$ & $\mathrm{I}_{3}$ & $\mathrm{I}_{3}$ & $2 \mathrm{I}_{3}$ & $2 \mathrm{I}_{3}$ & linear & linear \\
& & $(16)$ & $(16)$ & $(32)$ & $(32)$ & & \\
$| \pm 1, \pm 1, \pm 1\rangle$ & $\mathrm{E}_{2}$ & $\mathrm{E}_{2}$ & $\mathrm{E}_{2}$ & $\mathrm{E}_{3}+\mathrm{E}_{4}$ & $2 \mathrm{E}_{2}$ & linear & quad \\
& & $(8)$ & $(8)$ & $(4+4)$ & $(16)$ & & \\
\hline
\end{tabular}

a Statistical weights here differ from those in Ref. [13]. Our results agree with the $\Gamma_{\text {spin }}$ decomposition and with Table II in Ref. [13], but we have counted as Pauli-allowed all torsion.rotation-nuclear-spin states of species ${ }^{t r n} A^{1}$ and ${ }^{t r n} A_{2}$ formed in direct products of the form ${ }^{t r} \Gamma \times{ }^{n} \Gamma$.

${ }^{b}$ Four more torsional basis functions must be generated for each of the four $I$ species rows by cyclic permutation of the quantum numbers inside the ket.

with the statistical weights for their rotational levels [13]. The symmetries of the symmetric top wavefunctions $|J K M\rangle$ are $A_{1}$ and $A_{2}$ for $J$ even and odd, respectively, when $K=0 ; A_{1} \oplus A_{2}$ when $K=3 n>0$; and $E_{1}$ when $K=3 n \pm 1$. The rotational levels of $I_{2}$ torsional states have the largest statistical weight and can be expected to be dominant in the spectrum. 


\subsection{First-order Coriolis coupling}

It is well known from earlier studies that for the problem of an asymmetric top molecule containing one methyl rotor, torsion-rotation levels of the totally symmetric component $A_{1}$ can always be fitted using a rigid rotor Hamiltonian to obtain effective rotational constants. But it has not been explicitly shown yet if this also applies to a $\mathrm{C}_{3 v}$-symmetric molecule of the type $\left(\mathrm{CH}_{3}\right)_{3} \mathrm{XY}$, for which additional degeneracies are introduced by its high symmetry. To describe rotational levels of a given torsional state using a rigid rotor Hamiltonian, the matrix elements for first-order Coriolis coupling between torsion and overall rotation must vanish. This in turn requires

$$
\left\langle n_{\Gamma}\left|p_{\alpha_{i}}\right| n_{\Gamma}^{\prime}\right\rangle=0,
$$

with $n_{\Gamma}$ and $n_{\Gamma}^{\prime}$ being torsional components of a state of symmetry $\Gamma$, and $p_{\alpha_{i}}$ being the torsional angular momentum of top $i(i=0,1,2)$. For $\left(\mathrm{CH}_{3}\right)_{3} \mathrm{SnCl}$ the three $p_{\alpha_{i}}$ span the symmetry species $A_{2} \oplus E_{1}$, and each $p_{\alpha_{i}}$ changes sign under time inversion. Watson [18] worked out a general theorem in which he shows that for operators $O$ changing sign under time inversion the matrix element $\left\langle n_{\Gamma}|O| n_{\Gamma}^{\prime}\right\rangle$ always vanishes if $\Gamma(O)$ does not belong to the representations of the antisymmetrized product $\left[\Gamma^{2}(n)\right]_{\text {antisym }}$ of the considered symmetry species. A direct product of an irreducible representation with itself can always be reduced into a symmetrized and an antisymmetrized product [19].

To obtain vanishing matrix elements for Coriolis coupling neither $A_{2}$ nor $E_{1}$ may be contained in the antisymmetrized product of the symmetry species of the torsional state. Applying this theorem to $\left(\mathrm{CH}_{3}\right)_{3} \mathrm{SnCl}$, i.e. the PI group $\mathrm{G}_{162}$, shows that indeed rotational levels of the $A_{1}$ and $A_{2}$ torsional states can be fitted using a rigid rotor Hamiltonian, while all others will be perturbed by first-order Coriolis coupling.

\subsection{High-barrier tunneling-rotation formalism}

To determine the relative ordering and to facilitate an assignment of the torsional transitions observed in the spectrum we used the high-barrier group-theoretical tunneling-rotation formalism [20] appropriate for $\mathrm{G}_{162}$, based on the coordinates and their transformation properties defined above, to predict the torsional splitting pattern for $K=0$. For a $\mathrm{C}_{3 v}$-symmetric molecule with three group-theoretically equivalent internal rotors such as $\left(\mathrm{CH}_{3}\right)_{3} \mathrm{SnCl}, n=27$ different frameworks are present. These frameworks can be generated by applying the $G_{162}$ operators $\alpha^{p} \beta^{q} \gamma^{r}$ to framework 1 (in Figure 2), with the integers $p, q$ and $r$ in the range $0 \leq p, q, r \leq 2$. 
The frameworks can be ordered in a number of ways, but for the purposes of this work we use the following numbering. Framework 1 has $p q r=000$. Frameworks $2-7$ are formed from framework 1 by rotating only one methyl group at a time and have $p q r=100,200,010,020,001,002$, respectively. Frameworks 8-13, formed from framework 1 by an antigeared rotation of two methyl groups, have $p q r=110,220,101,202,011,022$. Frameworks 14-19, formed by a geared rotation of two methyl groups, have $p q r=$ $120,210,102,201,012,021$. Frameworks 20 and 21, formed by a rotation of all three methyl groups in the same sense, have $p q r=111$ and 222. Frameworks 22-27, formed from framework 1 by a rotation of all three methyl groups, two in one sense and one in the other, have $p q r=112,221,121,212,211,122$.

These 27 frameworks can be interconnected by 351 tunneling pathways, represented mathematically by tunneling matrix elements. If the initial framework is taken to be framework 1, as displayed in Figure 2, then the 26 pathways and the corresponding matrix elements can be denoted as $1 \rightarrow n$ and $H_{1, n}$, respectively, $n=2-27$. The properties of the group $G_{162}$ can be used to show that only five of all tunneling pathways are topologically inequivalent. These five pathways and the corresponding five tunneling matrix elements (real for the $K=0$ problem) correspond physically to the ordering described above, i.e.: i) rotation of only one $\mathrm{CH}_{3}$ group $\left(H_{R}=H_{1, n}\right.$, $n=2-7)$, ii) rotation of two $\mathrm{CH}_{3}$ groups in a geared $\left(H_{G}=H_{1, n}, n=14-19\right)$ or antigeared way $\left(H_{A}=H_{1, n}, n=8-13\right)$; and iii) rotation of all three $\mathrm{CH}_{3}$ groups at the same time, either having the same $\left(H_{E}=H_{1, n}, n=20-21\right)$ or opposite sense $\left(H_{L}=H_{1, n}, n=22-27\right)$. In the high-barrier tunneling-rotation formalism it is necessary to assume that $\left(\mathrm{CH}_{3}\right)_{3} \mathrm{SnCl}$ spends most of its time vibrating in the vicinity of one of these 27 frameworks and only occasionally tunnels from one conformation to another [21]. Quantum mechanically speaking, we require the splittings caused by tunneling motions to be small compared to the vibrational spacings associated with a single equilibrium configuration (framework).

Based on our $a b$ initio calculations the distance of closest approach for two hydrogen atoms on different methyl groups is about $3.8 \AA$ for the equilibrium geometry of $\left(\mathrm{CH}_{3}\right)_{3} \mathrm{SnCl}$ (Figure 2), while in the equilibrium geometry of $\left(\mathrm{CH}_{3}\right)_{3} \mathrm{SiCl}[1,4]$ and $\left(\mathrm{CH}_{3}\right)_{3} \mathrm{GeCl}[3]$ the distances decrease to about $3.2 \AA$ and $3.3 \AA$, respectively. The van-der-Waals radius for hydrogen is $1.2 \AA$ indicating clearly that all five tunneling pathways have to be considered as feasible for $\left(\mathrm{CH}_{3}\right)_{3} \mathrm{SnCl}$. This leads to a tunneling Hamiltonian matrix for $K=0$ connecting all 27 frameworks by the five feasible tunneling pathways [4]. (Note that the definitions of the matrix elements $H_{L}$ and $H_{E}$ in the Supporting Information for Ref. [4] are exchanged with respect to those used here and in Ref. [3].) Diagonalization of this symmetric tunneling 


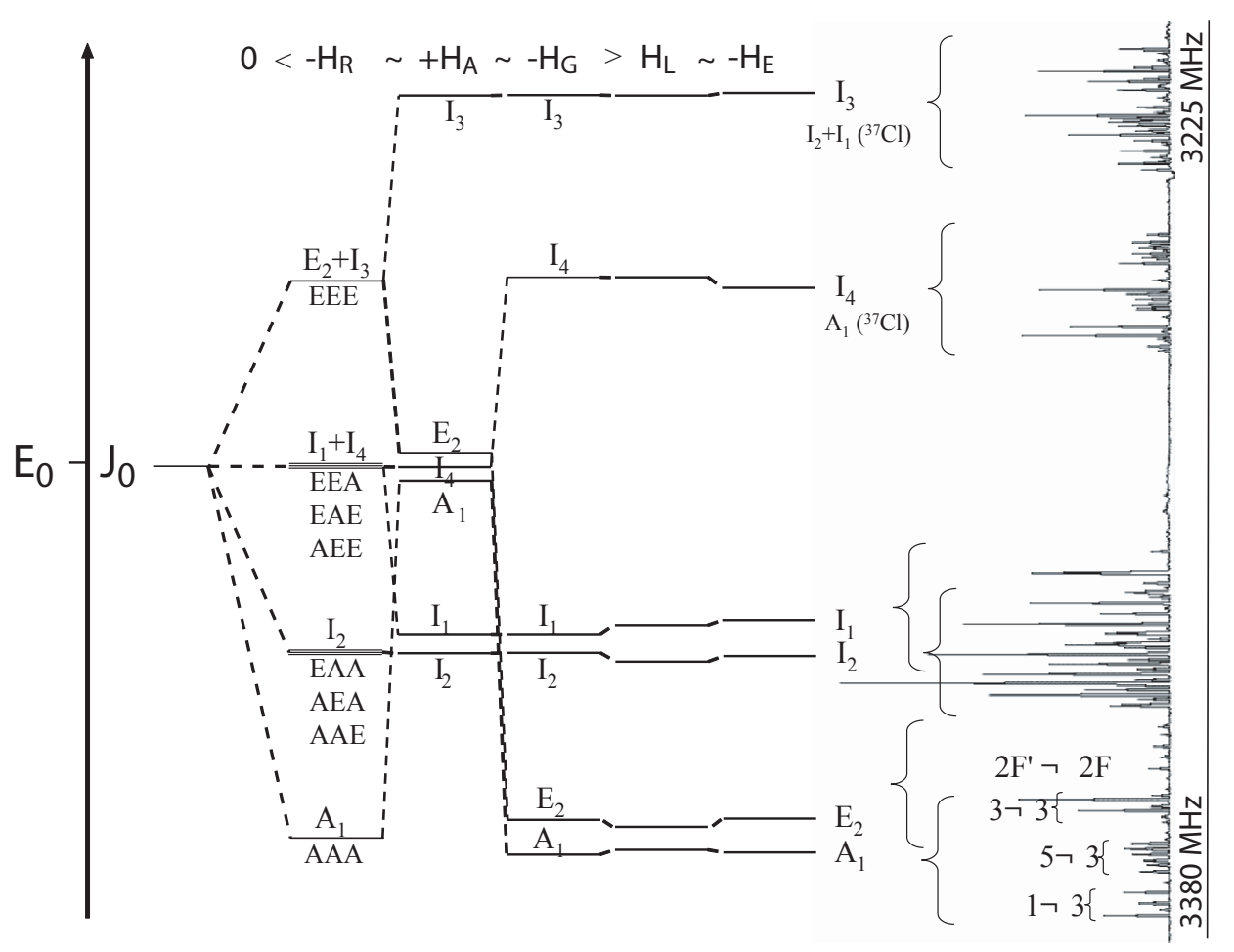

Figure 5. Torsional splitting pattern for the $J_{K}=J_{0}$ rotational energy level obtained from the high-barrier torsion-rotation tunneling formalism. The theoretical predictions are compared with the measured rotational spectrum for the $J+1 \leftarrow J=1 \leftarrow 0$-transition of the ${ }^{35} \mathrm{Cl}$ isotopologue (right). (The two regions of ${ }^{37} \mathrm{Cl}$ transitions occurring in the spectrum on the right should be ignored for this comparison.) Lines in the torsion-rotation symmetry groups are further split due to tin isotopologues and chlorine nuclear quadrupole hyperfine structure. Some of these quadrupole assignments are indicated here for the $A_{1}$ - and $E_{2}$-symmetry species groups.

matrix yields energy level expressions for the various symmetry species [4],

$$
\begin{aligned}
& W\left(A_{1}\right)=E_{0}+6 H_{R}+6 H_{A}+6 H_{G}+6 H_{L}+2 H_{E} \\
& W\left(E_{2}\right)=E_{0}-3 H_{R}-3 H_{A}+6 H_{G}-3 H_{L}+2 H_{E} \\
& W\left(I_{1}\right)=E_{0}-3 H_{A}+3 H_{L}-H_{E} \\
& W\left(I_{2}\right)=E_{0}+3 H_{R}-3 H_{L}-H_{E} \\
& W\left(I_{3}\right)=E_{0}-3 H_{R}+3 H_{A}-H_{E} \\
& W\left(I_{4}\right)=E_{0}-3 H_{G}+2 H_{E}
\end{aligned}
$$

and the torsion-rotation splitting pattern for $K=0$ displayed in Figure 5. This diagram is most easily understood by reading from left to right, starting with a $J_{K}=J_{0}=$ even symmetric rotor energy level at energy $E_{0}$. By considering internal rotation of only one methyl top at a time $\left(H_{1, n=2-7}=H_{R}<0\right.$, other $\left.H_{1, n}=0\right)$ the $J_{0}$-energy level splits into four torsion-rotation sublevels: $A_{1}, I_{2}, I_{1} \oplus I_{4}$ and $I_{3} \oplus E_{2}$, with $I_{1}$ and $I_{4}$ as well as $E_{2}$ and $I_{3}$ being degenerate. 
Note, that this pattern corresponds exactly to the one obtained using local mode theory [4].

As the next step we consider further modifications of the energy levels, which result when interactions due to internal rotation of two tops (geared $\left(H_{G}\right)$ and anti-geared $\left.\left(H_{A}\right)\right)$ as well as of three tops (with the same $\left(H_{E}\right)$ and opposite $\left(H_{L}\right)$ sense of rotation) are also included. Figure 5 thus indicates that the $J_{0}$ symmetric rotor level splits finally into six $G_{162}$ (see Table 1) torsion-rotation components $A_{1}, E_{2}, I_{1}, I_{2}, I_{3}$ and $I_{4}$ for $J_{\text {even }}$. For $J_{\text {odd }}$, these species must be multiplied by $\mathrm{A}_{2}$ to obtain $A_{2}, \mathrm{E}_{2}, \mathrm{I}_{1}, \mathrm{I}_{2}, \mathrm{I}_{3}$ and $\mathrm{I}_{5}$.

Based on other internal rotation studies we expect the $K=0$ pure rotational spectrum to mimic the $K=0$ torsional splitting pattern, i.e., to consist of six components with a relative spacing similar to that calculated for the energy levels, but with the opposite frequency ordering. (For example, we expect the torsional transition $A_{2} \leftarrow A_{1}\left(J_{\text {even }}\right)$ to be located at the high frequency end of the $K=0$ series in Figure 5). We display a part (between $3225-3380 \mathrm{MHz}$ ) of the recorded $J+1 \leftarrow J=1 \leftarrow 0$-transition, which only consists of $K=0$-transitions, on the right of Figure 5 to show that this expectation is fulfilled. The torsion-rotation groups, which exhibit nuclear quadrupole and isotopolog structures, are marked with braces and labeled by the corresponding symmetry species for $J=0$. For the $A_{1}$ torsional transition the quantum numbers indicate the different quadrupole coupling components $\left(2 F^{\prime} \leftarrow 2 F\right)$ of the transition. Each of these quadrupole components occurs for each of the seven tin isotopologues within one torsion-rotation group. Here it is interesting to point out that for the $J+1 \leftarrow J=1 \leftarrow 0$ transition the splitting due to quadrupole hyperfine coupling is larger than the separation between the different tin isotopologues. This is reversed for the higher $J$-transitions. Three torsion-rotation transitions of $\left(\mathrm{CH}_{3}\right)_{3} \mathrm{Sn}^{37} \mathrm{Cl}$, namely $A_{2} \leftarrow A_{1}, I_{1} \leftarrow I_{1}$, and $I_{2} \leftarrow I_{2}$, are also present in Figure 5 . However, the spectra of $\left(\mathrm{CH}_{3}\right)_{3} \mathrm{Sn}^{35} \mathrm{Cl}$ and $\left(\mathrm{CH}_{3}\right)_{3} \mathrm{Sn}^{37} \mathrm{Cl}$ are overlapped for the $J+1 \leftarrow J=1 \leftarrow 0$-transition only, while they are well separated for all higher $J$-transitions.

In order to obtain semiquantitative agreement between the theoretically derived splitting pattern of the torsion-rotation energy levels and the pattern of the observed torsion-rotation transition frequencies, the contributions of the five different torsional tunneling pathways have to be considered as follows:

$$
-H_{R} \approx-H_{G} \approx+H_{A}>-H_{E} \approx+H_{L}>0 \text {. }
$$

This means that $B$-value changes caused by torsional tunneling effects must be approximately the same for internal rotation of one $\left(H_{R}\right)$ and of two $\mathrm{CH}_{3}$ groups $\left(H_{A}, H_{G}\right)$, but with a different sign for $H_{A}$ (antigeared rotation of two $\mathrm{CH}_{3}$ groups at a time). The contributions of internal rotations of three methyl 
groups $\left(H_{E}, H_{L}\right)$ are, as expected, significantly smaller by about one order of magnitude. As a comparison, for $\left(\mathrm{CH}_{3}\right)_{3} \mathrm{SiCl}$ [1] we have to assume [4]

$$
-H_{R}>-H_{G} \approx+H_{A} \approx+H_{E} \approx-H_{L} \approx 0,
$$

with $\left|H_{R}\right|$ being much larger than the magnitudes of the other four. This change can be explained to zeroth order by the larger steric repulsion in $\left(\mathrm{CH}_{3}\right)_{3} \mathrm{SiCl}$ compared to $\left(\mathrm{CH}_{3}\right)_{3} \mathrm{SnCl}$ such that, from an energetic point of view, internal rotation of two and three methyl groups at a time is quite unlikely for $\left(\mathrm{CH}_{3}\right)_{3} \mathrm{SiCl}[4]$.

\section{Stark-Effect Measurements}

To further confirm our initial symmetry-species assignments from the highbarrier tunneling-rotation formalism of the $K=0$ torsion-rotation transitions (Figure 5) we performed Stark-effect measurements using the CAESAR arrangement [9] for the $J+1 \leftarrow J=1 \leftarrow 0$ - and $5 \leftarrow 4$-transitions to differentiate between torsion-rotation transitions on the basis of their different (i.e., linear vs. quadratic) Stark effect. Watson [18] deduced a strict selection rule for linear Stark-effect based on group-theoretical considerations

$$
\Gamma\left(\mu_{f}\right)=\Gamma(J \cdot \mu) \subset\left[\Gamma^{2}\right]_{\text {antisym }}{ }^{\prime}
$$

with $\Gamma\left(\mu_{f}\right)=A_{2}$ (symmetry species of the space-fixed dipole moment operator $\mu_{f}$ ). Eq. 12 is fulfilled when $A_{2}$ is contained in the antisymmetrized product of the symmetry species of the considered torsion-rotation state. If this is the case, the transitions involving torsion-rotation states of that symmetry can show a linear Stark-effect. Otherwise, the series expansion for Stark-splittings in powers of the electric field strength $E$ must begin with a quadratic term. The latter turns out to be the case for $A_{1}, A_{2}, I_{4}$, and $I_{5}$. For torsion-rotation levels of all other symmetries in $G_{162}$, the expansion may begin with a linear term in $E$. The group-theoretical results are included in Table 3 together with the experimentally observed Stark behavior of the six internal rotation groups ( $K=0$ only) of the $1 \leftarrow 0$-transition. As can be seen, the Stark measurements support our initial assignments based on the predicted splitting patterns (Figure 5).

Figure 6 shows example spectra for linear Stark behavior of the species $I_{2} \leftarrow I_{2}(J+1 \leftarrow J=5 \leftarrow 4$-transition, $K=1)$ at two different electric field strengths: $1.6 \mathrm{~V} / \mathrm{cm}$ (left panel) and $8.1 \mathrm{~V} / \mathrm{cm}$ (right panel). These spectra demonstrate that the Stark-shifted and Stark-split lines from our CAESAR arrangement are very narrow. Also, significant line splittings are already 


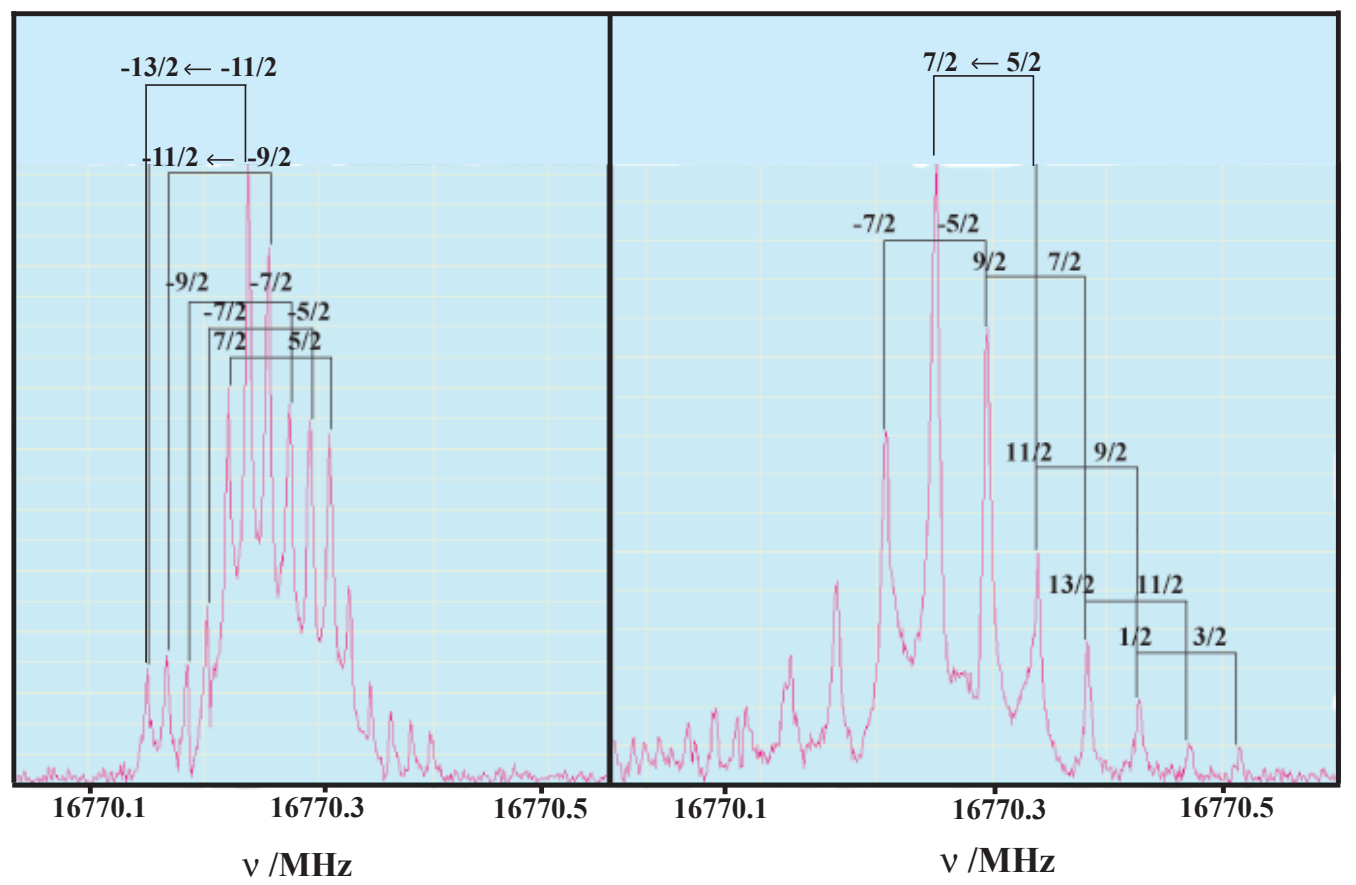

Figure 6. Amplitude spectra of the linearly Stark split torsion-rotation transition of the main isotopologue with $I_{2} \leftarrow I_{2}$ and $J+1 \leftarrow J=5 \leftarrow 4(K=1)$, shown at two different electric field strengths: $1.6 \mathrm{~V} / \mathrm{cm}$ (left panel) and $8.1 \mathrm{~V} / \mathrm{cm}$ (right panel). To avoid clutter, transitions with positive $M_{J}$ are labeled in the right panel, transitions with negative $M_{J}$ in the left.

observed at a relatively low electric field strength of only $1.6 \mathrm{~V} / \mathrm{cm}$, indicating a large dipole moment for $\left(\mathrm{CH}_{3}\right)_{3} \mathrm{SnCl}$.

Interestingly, not only $A_{2} \leftarrow A_{1}$ and $I_{5} \leftarrow I_{4}\left(J_{\text {even }}\right)$, but also the $E_{2} \leftarrow E_{2}$ torsion-rotation transition, which is located next to $A_{2} \leftarrow A_{1}$ in the spectrum (Figure 5), shows a quadratic Stark effect, even though, based on group theory, it could exhibit linear Stark behavior (Table 3). Furthermore, the experimental Stark-coefficients for the $A_{1}$ - and the $E_{2}$-species are absolutely identical.

As stated, we observed the unusual phenomenon of linear Stark effect for $K=0$ torsion-rotation transitions of a symmetric top molecule. The linear Stark effect is group-theoretically allowed for the $K=0$ torsion-rotation species $I_{1}, I_{2}, I_{3}$, and $E_{2}$ as given in Table 3 , while $A_{1}$ and $I_{4}\left(J_{\text {even }}\right)$ can only have quadratic Stark effect. Experimentally, however, we observe a quadratic Stark effect for $E_{2}$ (Table 3). Furthermore, the experimental Starkcoefficients for the $A_{1}$ and the $E_{2}$ species are absolutely identical. It seems possible that the linear Stark effect for $K=0$ torsion-rotation species is due to $\Delta K= \pm 1$ mixing between the $K=1$ and $K=0$ sublevels, since only torsional states which have the same symmetry species for $K=0$ and 
$K=1$ show linear Stark effect. Usually, $\Delta K= \pm 1$ mixing plays no role for the ground vibrational state of symmetric top molecules. For $\left(\mathrm{CH}_{3}\right)_{3} \mathrm{SnCl}$ it is possible that coupling of the internal rotation angular momenta of the three equivalent methyl groups with the overall angular momentum leads to these mixing effects. In addition, $\left(\mathrm{CH}_{3}\right)_{3} \mathrm{SnCl}$ loses its $\mathrm{C}_{3 v}$ equilibrium symmetry when internal rotation takes place. To examine the assumption of $\Delta K= \pm 1$-mixing we set up a simple wave function linear combination (LC) ansatz of the $|J K M\rangle=|J 1 M\rangle$ and $|J 0 M\rangle$ states

$$
\left|\Psi_{L C}\right\rangle=\left|\alpha \cdot \psi_{a}(K=0)+\beta \cdot \psi_{b}(K=1)\right\rangle
$$

with the coefficients $\alpha$ and $\beta\left(\alpha^{2}+\beta^{2}=1\right)$, which describe the contributions of $K=0$ and $K=1$ to $\Psi_{L C}$. The $\Delta J=\Delta K=0$-matrix elements of the linear Stark effect operator can be written as

$$
H_{\text {Stark }}=-\frac{\mu E K}{(J+1)}
$$

for $M_{F}=F=I+J$, with $\mu$ as dipole moment and $E$ as electric field strength [11]. Following this approach we used our Stark effect measurements to determine the $K=1$ contribution in Eq. 13. For the $I_{1}$ and the $I_{2}$ species we found values of $\beta\left(I_{1}\right)=0.1$ and $\beta\left(I_{2}\right)=0.09$, respectively, with $\alpha^{2}+$ $\beta^{2}=1$. Consequently, $\Delta K= \pm 1$ mixing is quite small, but large enough to significantly change the physical picture of the Stark effect.

Stark effect measurements for other $\mathrm{M}$ states are complicated in the presence of a quadrupole nucleus such as chlorine. With the electric field strengths possible in the CAESAR arrangement and with quadrupole coupling splittings on the order of $0.5-2 \mathrm{MHz}$ for $\left(\mathrm{CH}_{3}\right)_{3} \mathrm{SnCl}$ we have to work in the inconvenient intermediate field regime, for which simplified methods of Stark analysis, which are useful for the weak field case, lose their applicability. To quantitatively analyse our Stark effect measurements, we used the program QSTARK [22], which diagonalizes the energy matrix separately for each value of the applied electric field.

To determine the dipole moment of $\left(\mathrm{CH}_{3}\right)_{3} \mathrm{SnCl}$ we concentrated only on the $J+1, \Gamma^{\prime} \leftarrow J, \Gamma=1, A_{2} \leftarrow 0, A_{1}$ torsion-rotation transition to avoid perturbations and influences from internal rotation. We were able to fit 28 lines, corresponding to five $2 M_{F}+2 \leftarrow 2 M_{F}$-quadrupole transitions (5 $\leftarrow 3$ and $3 \leftarrow 1$ for $2 F+2 \leftarrow 2 F=5 \leftarrow 3 ; 3 \leftarrow 1$ and $1 \leftarrow 3$ for $2 F+2 \leftarrow 2 F=$ $3 \leftarrow 3$, and $1 \leftarrow 3$ for $2 F+2 \leftarrow 2 F=1 \leftarrow 3$, respectively) using QSTARK. This fit gave a standard deviation of $\sigma=0.71 \mathrm{kHz}$ and a dipole moment of $\mu=3.4980(30) \mathrm{D}$ for $\left(\mathrm{CH}_{3}\right)_{3} \mathrm{SnCl}$. The calculated value of $\mu=3.318 \mathrm{D}$ (BP86/def-TZVPP/ecp-46-mwb(Sn)) is close to the experimental one. 
Table 4

Effective asymmetric-semi-rigid rotor molecular constants from a fit of the $A_{2} \leftarrow A_{1}$ ( $J$ =even) and $A_{1} \leftarrow A_{2}(J=$ odd $) J+1 \leftarrow J$ torsion-rotation transitions, i.e., from a fit of all A species transitions together, of different isotopologues of $\left(\mathrm{CH}_{3}\right)_{3} \mathrm{SnCl}$. $\mathrm{N}$ denotes the number of lines included in the fit, $\sigma$ gives the standard deviation of each semi-rigid-rotor fit, numbers in parentheses are standard deviations of the molecular parameters ${ }^{a}$ as determined from the fit.

\begin{tabular}{cccccc}
\hline \hline isotopologue & $B_{\text {eff }} / \mathrm{MHz}$ & eqQ/MHz & $D_{J} / \mathrm{kHz}$ & $\mathrm{N}$ & $\sigma / \mathrm{kHz}$ \\
\hline$\left(\mathrm{CH}_{3}\right)_{3}{ }^{120} \mathrm{Sn}^{35} \mathrm{Cl}$ & $1680.040124(72)$ & $-35.7747(22)$ & $0.3437(17)$ & 29 & 1.5 \\
$\left(\mathrm{CH}_{3}\right)_{3}{ }^{118} \mathrm{Sn}^{35} \mathrm{Cl}$ & $1680.94621(16)$ & $-35.7840(47)$ & $0.3466(28)$ & 22 & 2.2 \\
$\left(\mathrm{CH}_{3}\right)_{3}{ }^{116} \mathrm{Sn}^{35} \mathrm{Cl}$ & $1681.871339(59)$ & $-35.7704(28)$ & $0.3482(11)$ & 18 & 0.7 \\
$\left(\mathrm{CH}_{3}\right)_{3}{ }^{120} \mathrm{Sn}^{37} \mathrm{Cl}$ & $1634.19255(14)$ & $-28.2109(15)$ & $0.34^{b}$ & 3 & 0.5 \\
\hline \hline
\end{tabular}

${ }^{a}$ One standard uncertainty $(1 \sigma)$ in units of the last significant digit, as obtained from the least squares fitting (type A uncertainty, $k=1$ )[24].

${ }^{b}$ Fixed in the least squares fit.

\section{Spectral analyses and discussion}

\subsection{Rigid rotor symmetric top analysis}

As shown in Section 4.3, matrix elements for first order Coriolis coupling vanish only for $A_{1}$ and $A_{2}$ torsion-rotation transitions, implying that only they can be fitted to effective molecular constants using a rigid symmetric rotor Hamiltonian (including centrifugal distortion). As a starting point of our analysis, we thus performed such fits for three different isotopologues of the $A_{2} \leftarrow A_{1}$ (J=even) and $A_{1} \leftarrow A_{2}(J=$ odd) torsion-rotation transitions for six $J+1 \leftarrow J$-transitions $(J=0, \ldots, 5)$, explicitly taking the quadrupole components into account. We used the program SYM2QS [23], which is suited for linear and symmetric top molecules with up to two quadrupole nuclei. We assume an uncertainty in the frequency determination of the lines of $2 \mathrm{kHz}$.

The results for the different isotopologues are summarized in Table 4. For the main isotopologue $\left(\mathrm{CH}_{3}\right)_{3}{ }^{120} \mathrm{Sn}^{35} \mathrm{Cl}$ we included 29 lines in the fit and obtained an effective rotational constant $B=1680.040124(72) \mathrm{MHZ}$ and a quadrupole coupling constant $e q Q\left({ }^{35} \mathrm{Cl}\right)=-35.7747(22) \mathrm{MHZ}$. The standard deviation of this fit is $\sigma=1.5 \mathrm{kHz}$. The quadrupole coupling constants agree within twice their standard deviations for the three most abundant Snspecies of the ${ }^{35} \mathrm{Cl}$ isotopologue, while the effective $B$ constants increase with decreasing mass of the Sn-isotope by about $0.9 \mathrm{MHz}$, for each step from ${ }^{120} \mathrm{Sn}$ to ${ }^{118} \mathrm{Sn}$ to ${ }^{116} \mathrm{Sn}$ (Table 4). Because of overlapping lines and lower intensities, the number of lines included in the fit becomes smaller for the 
weaker isotopologues. The spectrum of the ${ }^{120} \mathrm{Sn}^{37} \mathrm{Cl}$ isotopologue was in fact too weak and overlapped to permit secure identification of the higher $\mathrm{J}$ transitions, so only the results of a two parameter fit (i.e., B and eqQ) of the $J+1 \leftarrow J=1 \leftarrow 0$ hyperfine components is presented. Nevertheless, the ratio of the quadrupole coupling constants for chlorine determined from Table 4 for the ${ }^{120} \mathrm{Sn}$ isotopologues, $\mathrm{Q}\left({ }^{35} \mathrm{Cl}\right) / \mathrm{Q}\left({ }^{37} \mathrm{Cl}\right)=1.2681(1)$, is very close to the atomic quadrupole moment ratio of 1.2688 [11]. Table 5 gives the $A_{1} / A_{2}$ torsion-rotation transition frequencies included in the SYM2QS fits for the different isotopologues.

Table 5: Torsion-rotation transition frequencies $(K=0$; $A_{2} \leftarrow A_{1}(J=$ even $), A_{1} \leftarrow A_{2}(J=$ odd $\left.)\right)$ for different isotopologues of $\left(\mathrm{CH}_{3}\right)_{3} \mathrm{SnCl}$ with $v_{o b s}$ : observed frequency, $v_{\text {calc }}$ : calculated frequency from the SYM2QS least-square fit, and $\delta v=v_{o b s}-v_{\text {calc }}$.

\begin{tabular}{|c|c|c|c|c|c|c|c|}
\hline isotopologue & $J^{\prime}$ & $J$ & $2 F^{\prime}$ & $2 F$ & $v_{\text {calc }} / \mathrm{MHz}$ & $v_{o b s} / \mathrm{MHz}$ & $\delta v / \mathrm{kHz}$ \\
\hline \multirow[t]{18}{*}{$\left(\mathrm{CH}_{3}\right)_{3}{ }^{120} \mathrm{Sn}^{35} \mathrm{Cl}$} & 1 & 0 & 1 & 3 & 3369.0305 & 3369.0301 & -0.37 \\
\hline & & & 5 & 3 & 3361.8710 & 3361.8745 & 3.53 \\
\hline & & & 3 & 3 & 3352.9302 & 3352.9279 & -2.29 \\
\hline & 2 & 1 & 5 & 3 & 6720.9161 & 6720.9183 & 2.11 \\
\hline & & & 7 & 5 & 6720.9175 & 6720.9183 & 0.71 \\
\hline & & & 3 & 1 & 6711.2138 & 6711.2145 & 0.73 \\
\hline & & & 1 & 1 & 6720.1495 & 6720.1497 & 0.15 \\
\hline & & & 5 & 5 & 6711.9753 & 6711.9767 & 1.36 \\
\hline & 3 & 2 & 9 & 7 & 10080.6303 & 10080.6318 & 1.51 \\
\hline & & & 7 & 5 & 10080.6297 & 10080.6318 & 2.09 \\
\hline & & & 7 & 7 & 10071.6875 & 10071.6867 & -0.83 \\
\hline & 4 & 3 & 11 & 9 & 13440.5045 & 13440.5037 & -0.80 \\
\hline & & & 9 & 7 & 13440.5042 & 13440.5037 & -0.51 \\
\hline & & & 7 & 5 & 13439.4650 & 13439.4634 & -1.56 \\
\hline & & & 5 & 3 & 13439.4664 & 13439.4634 & -2.96 \\
\hline & & & 5 & 5 & 13448.4072 & 13448.4049 & -2.26 \\
\hline & & & 7 & 7 & 13443.6447 & 13443.6439 & -0.81 \\
\hline & & & 9 & 9 & 13431.5614 & 13431.5610 & -0.42 \\
\hline
\end{tabular}


Table 5: continue: Torsion-rotation transition frequencies $\left(K=0 ; A_{2} \leftarrow A_{1}(J=\right.$ even $), A_{1} \leftarrow A_{2}(J=$ odd $\left.)\right)$ for different isotopologues of $\left(\mathrm{CH}_{3}\right)_{3} \mathrm{SnCl}$.

\begin{tabular}{|c|c|c|c|c|c|c|c|}
\hline isotopologue & $J^{\prime}$ & $J$ & $2 F^{\prime}$ & $2 F$ & $v_{\text {calc }} / \mathrm{MHz}$ & $v_{o b s} / \mathrm{MHz}$ & $\delta v / \mathrm{kHz}$ \\
\hline & 5 & 4 & 13 & 11 & 16800.4173 & 16800.4178 & 0.49 \\
\hline & & & 11 & 9 & 16800.4172 & 16800.4178 & 0.65 \\
\hline & & & 9 & 7 & 16799.8027 & 16799.8028 & 0.11 \\
\hline & & & 7 & 5 & 16799.8033 & 16799.8028 & -0.47 \\
\hline & & & 9 & 9 & 16802.9432 & 16802.9455 & 2.22 \\
\hline & 6 & 5 & 15 & 13 & 20160.3223 & 20160.3229 & 0.51 \\
\hline & & & 13 & 11 & 20160.3222 & 20160.3229 & 0.61 \\
\hline & & & 11 & 9 & 20159.9130 & 20159.9134 & 0.32 \\
\hline & & & 9 & 7 & 20159.9133 & 20159.9134 & 0.03 \\
\hline & & & 13 & 13 & 20151.3790 & 20151.3792 & 0.20 \\
\hline & & & 11 & 11 & 20162.4391 & 20162.4379 & -1.19 \\
\hline \multirow[t]{3}{*}{$\left(\mathrm{CH}_{3}\right)_{3}{ }^{120} \mathrm{Sn}^{37} \mathrm{Cl}$} & 1 & 0 & 1 & 3 & 3275.4416 & 3275.4413 & -0.22 \\
\hline & & & 5 & 3 & 3269.7964 & 3269.7968 & 0.39 \\
\hline & & & 3 & 3 & 3262.7455 & 3262.7454 & -0.17 \\
\hline \multirow[t]{11}{*}{$\left.\left(\mathrm{CH}_{3}\right)_{3}\right)^{118} \mathrm{Sn}^{35} \mathrm{Cl}$} & 1 & 0 & 1 & 3 & 3370.8450 & 3370.8415 & -3.47 \\
\hline & & & 5 & 3 & 3363.6836 & 3363.6890 & 5.40 \\
\hline & & & 3 & 3 & 3354.7405 & 3354.7378 & -2.67 \\
\hline & 2 & 1 & 7 & 5 & 6724.5420 & 6724.5426 & 0.55 \\
\hline & & & 5 & 3 & 6724.5406 & 6724.5426 & 1.95 \\
\hline & 3 & 2 & 9 & 7 & 10086.0666 & 10086.0660 & -0.66 \\
\hline & & & 7 & 5 & 10086.0660 & 10086.0660 & -0.08 \\
\hline & & & 5 & 3 & 10083.8473 & 10083.8489 & 1.63 \\
\hline & & & 3 & 1 & 10083.8524 & 10083.8489 & -3.45 \\
\hline & 4 & 3 & 11 & 9 & 13447.7525 & 13447.7525 & -0.07 \\
\hline & & & 9 & 7 & 13447.7522 & 13447.7525 & 0.22 \\
\hline
\end{tabular}


Table 5: continue: Torsion-rotation transition frequencies $\left(K=0 ; A_{2} \leftarrow A_{1}(J=\right.$ even $), A_{1} \leftarrow A_{2}(J=$ odd $\left.)\right)$ for different isotopologues of $\left(\mathrm{CH}_{3}\right)_{3} \mathrm{SnCl}$.

\begin{tabular}{|c|c|c|c|c|c|c|c|}
\hline isotopologue & $J^{\prime}$ & $J$ & $2 F^{\prime}$ & $2 F$ & $v_{\text {calc }} / \mathrm{MHz}$ & $v_{o b s} / \mathrm{MHz}$ & $\delta v / \mathrm{kHz}$ \\
\hline & & & 9 & 9 & 13438.8071 & 13438.8086 & 1.43 \\
\hline & & & 7 & 7 & 13450.8935 & 13450.8939 & 0.35 \\
\hline & 5 & 4 & 13 & 11 & 16809.4768 & 16809.4787 & 1.90 \\
\hline & & & 11 & 9 & 16809.4766 & 16809.4787 & 2.07 \\
\hline & & & 9 & 7 & 16808.8620 & 16808.8596 & -2.43 \\
\hline & & & 7 & 5 & 16808.8626 & 16808.8596 & -3.01 \\
\hline & 6 & 5 & 15 & 13 & 20171.1929 & 20171.1933 & 0.34 \\
\hline & & & 13 & 11 & 20171.1928 & 20171.1933 & 0.45 \\
\hline & & & 11 & 9 & 20170.7835 & 20170.7838 & 0.32 \\
\hline & & & 9 & 7 & 20170.7838 & 20170.7838 & 0.02 \\
\hline & & & 13 & 13 & 20162.2472 & 20162.2466 & -0.63 \\
\hline \multirow[t]{14}{*}{$\left(\mathrm{CH}_{3}\right)_{3}{ }^{116} \mathrm{Sn}^{35} \mathrm{Cl}$} & 1 & 0 & 1 & 3 & 3372.6918 & 3372.6915 & -0.30 \\
\hline & 2 & 1 & 7 & 5 & 6728.2422 & 6728.2426 & 0.43 \\
\hline & & & 5 & 3 & 6728.2408 & 6728.2426 & 1.83 \\
\hline & 3 & 2 & 9 & 5 & 10091.6170 & 10091.6172 & 0.16 \\
\hline & & & 7 & 5 & 10091.6165 & 10091.6172 & 0.74 \\
\hline & 4 & 3 & 11 & 9 & 13455.1530 & 13455.1525 & -0.53 \\
\hline & & & 9 & 7 & 13455.1527 & 13455.1525 & -0.24 \\
\hline & & & 7 & 5 & 13454.1136 & 13454.1141 & 0.43 \\
\hline & & & 5 & 3 & 13454.1150 & 13454.1141 & -0.96 \\
\hline & & & 7 & 7 & 13458.2929 & 13458.2927 & -0.16 \\
\hline & 5 & 4 & 13 & 11 & 16818.7272 & 16818.7272 & -0.01 \\
\hline & & & 11 & 9 & 16818.7270 & 16818.7272 & 0.16 \\
\hline & & & 9 & 7 & 16818.1127 & 16818.1121 & -0.62 \\
\hline & & & 7 & 5 & 16818.1132 & 16818.1121 & -1.20 \\
\hline
\end{tabular}


Table 5: continue: Torsion-rotation transition frequencies $\left(K=0 ; A_{2} \leftarrow A_{1}(J=\right.$ even $), A_{1} \leftarrow A_{2}(J=$ odd $\left.)\right)$ for different isotopologues of $\left(\mathrm{CH}_{3}\right)_{3} \mathrm{SnCl}$.

\begin{tabular}{lccccccc}
\hline \hline isotopologue & $J^{\prime}$ & $J$ & $2 F^{\prime}$ & $2 F$ & $v_{\text {calc }} / \mathrm{MHz}$ & $v_{\text {obs }} / \mathrm{MHz}$ & $\delta v / \mathrm{kHz}$ \\
\hline & 6 & 5 & 15 & 13 & 20182.2930 & 20182.2935 & 0.44 \\
& & & 13 & 11 & 20182.2929 & 20182.2935 & 0.54 \\
& & & 11 & 9 & 20181.8837 & 20181.8840 & 0.25 \\
& & & 7 & 20181.8840 & 20181.8840 & -0.04 \\
\hline \hline
\end{tabular}

\subsection{Further discussion of the quadrupole coupling}

Comparing the quadrupole coupling constant of $\left(\mathrm{CH}_{3}\right)_{3} \mathrm{SnCl}\left(\mathrm{eqQ}\left({ }^{35} \mathrm{Cl}\right)=\right.$ $-35.776(81) \mathrm{MHZ}$ ) (obtained from the program XIAM [25], see below) with those of $\left(\mathrm{CH}_{3}\right)_{3} \mathrm{GeCl}[3,4]\left(e q Q\left({ }^{35} \mathrm{Cl}\right)=-40.0711(14) \mathrm{MHZ}\right),\left(\mathrm{CH}_{3}\right)_{3} \mathrm{SiCl}$ [1] $\left(e q Q\left({ }^{35} \mathrm{Cl}\right)=-34.81134(83) \mathrm{MHZ}\right)$ and $\left(\mathrm{CH}_{3}\right)_{3} \mathrm{CCl}$ [6] $\left(\mathrm{eqQ}\left({ }^{35} \mathrm{Cl}\right)=\right.$ $-67.312(3) \mathrm{MHZ})$, it can be seen that the absolute value for $\left(\mathrm{CH}_{3}\right)_{3} \mathrm{CCl}$ exceeds those for $\left(\mathrm{CH}_{3}\right)_{3} \mathrm{SnCl},\left(\mathrm{CH}_{3}\right)_{3} \mathrm{GeCl}$ and $\left(\mathrm{CH}_{3}\right)_{3} \mathrm{SiCl}$ by almost a factor of two. Using the model developed by Townes and Dailey [11], it is possible to obtain information on the character of the X-Cl bonds from the experimental coupling constants. This procedure is discussed in more detail in Ref. [4] and yields $35.4 \%$ ionic and $64.6 \%$ single $(\sigma)$ bond character for the $\mathrm{C}-\mathrm{Cl}$ bond, $19.8 \%$ ionic, $48.5 \%$ single $(\sigma)$ and $31.7 \%$ double $(\pi)$ bond character for the $\mathrm{Si}-\mathrm{Cl}$ bond, $37.6 \%$ ionic, $46.5 \%$ single and $15.9 \%$ double bond character for the $\mathrm{Ge}-\mathrm{Cl}$ bond, and $51.8 \%$ ionic, $39.5 \%$ single and $8.7 \%$ double bond character for the $\mathrm{Sn}-\mathrm{Cl}$ bond [4]. These results are in line with general knowledge about the bonding properties of $\mathrm{C}, \mathrm{Si}$ and $\mathrm{Sn}$ : Carbon is relatively compact and can only form single bonds in a fourfold bonding situation. In contrast, the formation of double bonds employing back bonding processes with empty $3 \mathrm{~d}$-orbitals is a well investigated phenomenon in silicon chemistry. As expected, the ionic character is largest for the $\mathrm{Sn}-\mathrm{Cl}$ bond.

As already mentioned in Section 3 and displayed in Figure 4c, we observed an anomalous $K=0$ quadrupole pattern for some torsion-rotation transitions, which depends also on $J$. For example, the two $J+1 \leftarrow J=5 \leftarrow 4$ quadrupole coupling components for $K=0$ with $F^{\prime} \leftarrow F=\frac{13}{2} \leftarrow \frac{11}{2}$ and $\frac{11}{2} \leftarrow \frac{9}{2}$ as well as the two with $\frac{9}{2} \leftarrow \frac{7}{2}$ and $\frac{7}{2} \leftarrow \frac{5}{2}$ usually form accidentally degenerate pairs in chlorine-containing $\left(I=\frac{3}{2}\right)$ symmetric top molecules such as $\left(\mathrm{CH}_{3}\right)_{3} \mathrm{SnCl}$. We find this accidental degeneracy for $A_{1}$ and $E_{2}$ torsion- 
rotation transitions, but not for $I_{1}, I_{2}, I_{3}$ and $I_{4}$. This observation is consistent with the assumption of $\Delta K= \pm 1$-mixing, which can be understood from the $K=0$ and $K=1$ symmetry species in Table 3 . The frequency differences between $\frac{13}{2} \leftarrow \frac{11}{2}$ and $\frac{11}{2} \leftarrow \frac{9}{2}$ as well as between $\frac{9}{2} \leftarrow \frac{7}{2}$ and $\frac{7}{2} \leftarrow \frac{5}{2}$ are in the range of $40-450 \mathrm{kHz}$, and depend on $J$ and on the torsional species (Table 6). If we again assume $K=1$ contributions of $\beta=0.1$ in Eqn. 13, splittings of this order of magnitude become possible. This can be shown by considering the interaction of the internal rotation of the three methyl groups with the quadrupole coupling. The appropriate totally symmetric and time-reversal invariant quadrupole coupling Hamiltonian for this problem is

$$
\begin{aligned}
H_{Q} & =[e Q / I(2 I-1) J(J+1)(2 J-1)(2 J+3)] \times\left[q_{-+}\left(\alpha_{0} \alpha_{1} \alpha_{2}\right)\left(J_{+} J_{-}+J_{-} J_{+}\right)\right. \\
& +q_{z z}\left(\alpha_{0} \alpha_{1} \alpha_{2}\right) J_{z}^{2}+q_{-z}\left(\alpha_{0} \alpha_{1} \alpha_{2}\right)\left(J_{+} J_{z}+J_{z} J_{+}\right)+q_{+z}\left(\alpha_{0} \alpha_{1} \alpha_{2}\right)\left(J_{-} J_{z}+J_{z} J_{-}\right) \\
& \left.+q_{--}\left(\alpha_{0} \alpha_{1} \alpha_{2}\right) J_{+}^{2}+q_{++}\left(\alpha_{0} \alpha_{1} \alpha_{2}\right) J_{-}^{2}\right] \times\left[3(\mathbf{I} \cdot \mathbf{J})^{2}+\frac{3}{2}(\mathbf{I} \cdot \mathbf{J})-I^{2} \cdot J^{2}\right]
\end{aligned}
$$

with $J$ being the overall angular momentum, $I$ being the nuclear angular momentum of the chlorine nucleus $(I=3 / 2), J_{z}$ being the projection of the overall angular momentum on the molecular z-axis (of symmetry $A_{2}$ in Table 1), and

$$
J_{ \pm}=J_{x} \pm i J_{y} \text {. }
$$

The operators $\pm J_{ \pm}$are of symmetry $\mathrm{E}_{1 \pm}$, where the \pm subscripts are defined such that

$$
c\left|E_{1 \pm}\right\rangle=\exp (\mp 2 \pi i / 3)\left|E_{1 \pm}\right\rangle
$$

and

$$
\sigma\left|E_{1 \pm}\right\rangle=+\left|E_{1 \mp}\right\rangle
$$

The $q$ 's are functions of the torsional angles $\alpha_{0}, \alpha_{1}$ and $\alpha_{2}$, chosen to have either $A_{1}$ or $E_{1}$ symmetry. Their subscripts imply transformation properties identical to those of products $J_{p} J_{q}+J_{q} J_{p}$ with the same subscripts. Eq. 15 shows that not only must the two $J$-operators of $\mathrm{A}_{1}$ symmetry $\left(J_{+} J_{-}+J_{-} J_{+}\right)$and $J_{z}^{2}$ be considered to form the complete totally symmetric Hamiltonian $H_{Q}$, but also two J-operators of $E_{1 \pm}$-symmetry, i.e., $\left(J_{ \pm} J_{z}+J_{z} J_{ \pm}\right)$and $J_{\mp}^{2}$, multiplied by appropriate $q$ 's of $E_{1 \mp}$ symmetry, must be included. These additional $J$-operators are responsible for $\Delta K= \pm 1$ and $\Delta K= \pm 2$ mixing, since they change the value of $K$.

To determine whether $\Delta K= \pm 1$-mixing is possible for a torsion-rotation state of given symmetry $\Gamma$ we qualitatively determined the corresponding matrix elements. As can be seen from Eqn. 15, each term in $H_{Q}$ can be separated into a rotational $(J, I)$ and a torsional (q) factor. This can also be assumed for the appropriate basis set for this problem

$$
\left|I J K F M_{F} v_{t} \Gamma\right\rangle=\left|I J K F M_{F}\right\rangle \cdot\left|v_{t} \Gamma\right\rangle,
$$


Table 6

Observed additional $K=0$ quadrupole splitting (in kHz) between the $F+1 \leftarrow F$ and $F \leftarrow F-1$ hyperfine components (as explained in the text) for different $J$-transitions and different torsional species of $\left(\mathrm{CH}_{3}\right)_{3}{ }^{120} \mathrm{Sn}^{35} \mathrm{Cl}$.

\begin{tabular}{cccccc}
\hline \hline$J$ & 1 & 2 & 3 & 4 & 5 \\
$F$ & $\frac{5}{2}$ & $\frac{7}{2}$ & $\frac{9}{2}$ & $\frac{11}{2}$ & $\frac{13}{2}$ \\
& & $\frac{3}{2}$ & $\frac{5}{2}$ & $\frac{7}{2}$ & $\frac{9}{2}$ \\
& & & & & \\
$\Gamma_{\text {torsional }}$ & & & & & \\
\hline$I_{2}$ & 82 & 86 & 68 & 53 & 40 \\
$I_{1}$ & & 99 & 75 & 57 & 43 \\
$I_{4}$ & 361 & 262 & 165 & 109 & 74 \\
$I_{3}$ & 453 & 331 & 200 & 123 & 82 \\
\hline \hline
\end{tabular}

with $\left|v_{t} \Gamma\right\rangle$ being a torsional wavefunction. Consequently, integrals for this problem can be split up into a torsion and a rotation-quadrupole part, with the torsion integral having one of the two forms

$$
\left\langle v_{t} \Gamma\left|q\left(A_{1}\right)\right| v_{t}^{\prime} \Gamma^{\prime}\right\rangle
$$

or

$$
\left\langle v_{t} \Gamma\left|q\left(E_{1 \mp}\right)\right| v_{t}^{\prime} \Gamma^{\prime}\right\rangle,
$$

where $q\left(A_{1}\right)$ stands for the $A_{1}$ and $q\left(E_{1 \mp}\right)$ for the $E_{1 \mp}$ symmetry $q$-functions, respectively. $\Delta K= \pm 1$-mixing within a given torsional state $\left(v_{t} \Gamma=v_{t}^{\prime} \Gamma^{\prime}\right)$, and thus additional quadrupole splitting is only possible if the symmetrized product of the torsional species with itself contains the symmetry species of the $q\left(E_{1 \mp}\right)$ functions [18]:

$$
\left[\Gamma^{2}\left(v_{t}\right)\right]_{s y m m} \supset E_{1}
$$

Eq. 22 is only fulfilled for torsional states of $E_{1}$ or I-symmetry, which is in agreement with our experimental observations for the torsional states in Figure 5. Furthermore, the size of the additional splitting decreases with increasing $J$-value, while it increases for the different torsion-rotation transitions in the order

$$
\Delta v_{\text {splitting }}\left(I_{2}\right)<\Delta v_{\text {splitting }}\left(I_{1}\right)<\Delta v_{\text {splitting }}\left(I_{4}\right)<\Delta v_{\text {splitting }}\left(I_{3}\right) \text {, }
$$

as summarized in Table 6 . We assume that this order reflects the amount of $\Delta K= \pm 1$-mixing for the different torsional species, which would then be largest for $I_{3}$ and smallest for $I_{2}$. This is in agreement with our findings from the $K=0$ Stark effect measurements, where the linear behavior is also ascribed to mixing with $K=1$ levels. 
We performed initial global fits of all six torsion-rotation transitions for $K=0$ for six different $J+1 \leftarrow J$-transitions $(J=0-5)$ using the program XIAM [25], which is suitable for molecules with up to three internal rotors including one quadrupole nucleus. The theoretical background on which this program is based is already discussed in the literature in several articles $[1,2,25]$ so that we will only review the structure of the Hamiltonian. It consists of different contributions

$$
H=H_{r o t}+H_{t o r s}+H_{r r}+H_{t t}+H_{r t}+H_{c d}+H_{n q}
$$

The first term represents the Hamiltonian of a rigid rotor consisting of a rigid frame and three equivalent internal rotors, the second term, $H_{\text {tors }}$, represents the Hamiltonian of a Mathieu oscillator. It describes the contribution of hindered internal rotation of the three methyl rotors. For a single rotor this is

$$
H_{\text {tors }}=F p_{1}^{2}+\frac{V_{3}}{2}\left(1-\cos 3 \alpha_{1}\right)
$$

$F$ may be considered as the effective rotational constant of the three equivalent internal rotors. $V_{3}$ is the threefold hindering potential and $\alpha_{1}$ the torsional angle of internal rotor 1 . The operator $H_{r r}$ is caused by the internal rotation and may be interpreted as a change of the location of the principal axes system due to the angular momentum created by the internal rotation. $H_{t t}$ describes the kinetic interaction between the internal rotors. The term $H_{r t}$ represents a Coriolis type interaction between the internal rotation and the overall rotation of the whole molecule. $H_{c d}$ contains the common quartic terms due to centrifugal distortion of a symmetric top,

$$
H_{c d}=-D_{J} J^{4}-D_{J K} J^{2} J_{z}^{2}-D_{K} J_{z}^{4}
$$

with the centrifugal distortion constants $D_{J}, D_{J K}$, and $D_{K}$. Since we restricted these fits to $K=0$-transitions, we consider only the first term of $H_{c d}$. Since the chlorine atomic nucleus has a quadrupole moment, the quadrupole operator $H_{n q}$ is also included in Eq. 24.

Table 7 gives the observed and calculated frequencies as well as their difference resulting from the least-square fit with XIAM of the $K=0$ transitions of the main isotopologue $\left(\mathrm{CH}_{3}\right)_{3}{ }^{120} \mathrm{Sn}^{35} \mathrm{Cl}$. Unc describes the experimental uncertainty of the frequency measurement. Due to overlapping lines or low intensity a few lines have been included in the fit with an uncertainty slightly higher than $2 \mathrm{kHz}$. The standard deviation is $\sigma=129 \mathrm{kHz}$, which is relatively high. Especially the torsional species $I_{3}$ and $I_{4}$ show deviations as large as 100 to $400 \mathrm{kHz}$ (Table 7). A fit without these torsional species has a much smaller uncertainty of $\sigma=21 \mathrm{kHz}$. These results indicate that 
$\Delta K= \pm 1$-mixing effects may not be completely accounted for in the XIAM program [25]. In the literature standard deviations up to $150 \mathrm{kHz}$ are not unusual for least-square XIAM fits of molecules with relatively large torsional splittings in the microwave spectrum. For $p$-fluorotoluene, for example, the $m=0$ and $m=1$ torsion-rotation transitions (108 lines) could be fitted to about $\sigma=27 \mathrm{kHz}$ using XIAM [26], while the $m=3$ torsion-rotation transitions (66 lines) have a standard deviation of $\sigma=162 \mathrm{kHz}$ [26]. Nevertheless, the XIAM fits were very useful for the global analysis of the rotational spectrum of $\left(\mathrm{CH}_{3}\right)_{3} \mathrm{SnCl}$, since they helped to initially assign the large number of torsion-rotation transitions. Furthermore, molecular parameters such as the rotational constant $B$ are determined to $4 \mathrm{kHz}$ (see also Table 8), and the barrier to internal rotation of the three methyl groups $V_{3}$ can be determined to $0.4 \mathrm{~J}$.

Table 7: Torsion-rotation transition frequencies $(K=0)$ of the main isotopologue $\left(\mathrm{CH}_{3}\right)_{3}{ }^{120} \mathrm{Sn}^{35} \mathrm{Cl}$ with $v_{\text {obs }}$ : observed frequency, $v_{\text {calc }}$ : calculated frequency from the least-square fit (XIAM), $\delta v: v_{o b s}-v_{c a l c}$. Unc gives the uncertainty of the line measurement from the experiment.

\begin{tabular}{|c|c|c|c|c|c|c|c|c|}
\hline$J^{\prime}$ & $J$ & $2 F^{\prime}$ & $2 F$ & Sym & $v_{\text {calc }} / \mathrm{GHz}$ & $\delta v / \mathrm{kHz}$ & $v_{o b s} / \mathrm{GHz}$ & $\mathrm{Unc}_{\mathrm{n}} \mathrm{kHz}$ \\
\hline \multirow[t]{15}{*}{1} & 0 & 5 & 3 & A1 & 3.3618942 & -19.7 & 3.3618745 & 2 \\
\hline & & 3 & 3 & A1 & 3.3529502 & -22.4 & 3.3529279 & 2 \\
\hline & & 1 & 3 & A1 & 3.3690493 & -19.2 & 3.3690301 & 2 \\
\hline & & 5 & 3 & E2 & 3.3536578 & 8.2 & 3.3536660 & 2 \\
\hline & & 3 & 3 & E2 & 3.3447139 & 11.2 & 3.3447251 & 2 \\
\hline & & 1 & 3 & E2 & 3.3608130 & 11.9 & 3.3608249 & 2 \\
\hline & & 5 & 3 & I1 & 3.3245049 & 6.1 & 3.3245110 & 2 \\
\hline & & 3 & 3 & I1 & 3.3161190 & 22.0 & 3.3161410 & 2 \\
\hline & & 1 & 3 & I1 & 3.3311995 & 7.9 & 3.3312073 & 2 \\
\hline & & 5 & 3 & $\mathrm{I} 2$ & 3.3277103 & 50.0 & 3.3277603 & 2 \\
\hline & & 3 & 3 & $\mathrm{I} 2$ & 3.3193012 & 59.5 & 3.3193607 & 2 \\
\hline & & 1 & 3 & I2 & 3.3344245 & 58.4 & 3.3344829 & 2 \\
\hline & & 5 & 3 & I3 & 3.2409897 & -409.0 & 3.2405808 & 2 \\
\hline & & 3 & 3 & I3 & 3.2336288 & -395.7 & 3.2332330 & 2 \\
\hline & & 1 & 3 & I3 & 3.2468495 & -415.3 & 3.2464342 & 2 \\
\hline
\end{tabular}


Table 7: continue: Torsion-rotation transition frequencies $(\mathrm{K}=0)$ of the main isotopologue $\left(\mathrm{CH}_{3}\right)_{3}{ }^{120} \mathrm{Sn}^{35} \mathrm{Cl}$.

\begin{tabular}{|c|c|c|c|c|c|c|c|c|}
\hline$J^{\prime}$ & $J$ & $2 F^{\prime}$ & $2 F$ & Sym & $v_{\text {calc }} / \mathrm{GHz}$ & $\delta v / \mathrm{kHz}$ & $v_{o b s} / \mathrm{GHz}$ & $U_{n c} / \mathrm{kHz}$ \\
\hline \multirow{26}{*}{2} & \multirow{26}{*}{1} & 5 & 3 & $\mathrm{I} 4$ & 3.2693932 & -100.9 & 3.2692923 & 2 \\
\hline & & 3 & 3 & $\mathrm{I} 4$ & 3.2617329 & -91.0 & 3.2616419 & 2 \\
\hline & & 1 & 3 & $\mathrm{I} 4$ & 3.2754962 & -103.8 & 3.2753924 & 2 \\
\hline & & 7 & 5 & A1 & 6.7209724 & -54.2 & 6.7209183 & 2 \\
\hline & & 5 & 3 & A1 & 6.7209724 & -54.2 & 6.7209183 & 2 \\
\hline & & 3 & 1 & A1 & 6.7112619 & -47.4 & 6.7112145 & 2 \\
\hline & & 1 & 1 & A1 & 6.7202058 & -56.2 & 6.7201497 & 2 \\
\hline & & 7 & 5 & E2 & 6.7045024 & 8.2 & 6.7045106 & 2 \\
\hline & & 5 & 3 & E2 & 6.7045024 & 8.2 & 6.7045106 & 2 \\
\hline & & 3 & 1 & E2 & 6.6947918 & 14.4 & 6.6948063 & 2 \\
\hline & & 3 & 3 & E2 & 6.7108909 & 15.2 & 6.7109061 & 2 \\
\hline & & 7 & 5 & $\mathrm{I} 1$ & 6.6529203 & -26.7 & 6.6528937 & 2 \\
\hline & & 5 & 3 & I1 & 6.6528240 & 69.7 & 6.6528937 & 2 \\
\hline & & 5 & 5 & I1 & 6.6444381 & 24.3 & 6.6444624 & 2 \\
\hline & & 3 & 1 & I1 & 6.6438027 & 29.0 & 6.6438317 & 2 \\
\hline & & 1 & 1 & I1 & 6.6522818 & 21.6 & 6.6523034 & 2 \\
\hline & & 5 & 5 & $\mathrm{I} 2$ & 6.6496975 & 105.5 & 6.6498030 & 2 \\
\hline & & 3 & 1 & $\mathrm{I} 2$ & 6.6490482 & 112.3 & 6.6491605 & 2 \\
\hline & & 1 & 1 & $\mathrm{I} 2$ & 6.6575354 & 103.7 & 6.6576391 & 2 \\
\hline & & 7 & 5 & I2 & 6.6581878 & 103.7 & 6.6582915 & 2 \\
\hline & & 5 & 3 & $\mathrm{I} 2$ & 6.6581066 & 102.5 & 6.6582091 & 2 \\
\hline & & 7 & 5 & I3 & 6.5299323 & -141.2 & 6.5297911 & 2 \\
\hline & & 5 & 3 & I3 & 6.5293972 & -59.2 & 6.5293380 & 2 \\
\hline & & 5 & 5 & I3 & 6.5220362 & -176.1 & 6.5218601 & 2 \\
\hline & & 3 & 1 & I3 & 6.5218171 & -152.1 & 6.5216651 & 2 \\
\hline & & 7 & 5 & I4 & 6.5687167 & -27.2 & 6.5686895 & 2 \\
\hline
\end{tabular}


Table 7: continue: Torsion-rotation transition frequencies $(K=0)$ of the main isotopologue $\left(\mathrm{CH}_{3}\right)_{3}{ }^{120} \mathrm{Sn}^{35} \mathrm{Cl}$.

\begin{tabular}{|c|c|c|c|c|c|c|c|c|}
\hline$J^{\prime}$ & $J$ & $2 F^{\prime}$ & $2 F$ & Sym & $v_{\text {calc }} / \mathrm{GHz}$ & $\delta v / \mathrm{kHz}$ & $v_{o b s} / \mathrm{GHz}$ & $U_{n c} / \mathrm{kHz}$ \\
\hline & & 5 & 3 & $\mathrm{I} 4$ & 6.5683390 & -10.5 & 6.5683285 & 2 \\
\hline & & 5 & 5 & $\mathrm{I} 4$ & 6.5606787 & -21.8 & 6.5606569 & 2 \\
\hline & & 3 & 1 & $\mathrm{I} 4$ & 6.5603177 & -12.8 & 6.5603050 & 2 \\
\hline & & 1 & 1 & $\mathrm{I} 4$ & 6.5683514 & -44.8 & 6.5683066 & 2 \\
\hline \multirow[t]{22}{*}{3} & 2 & 7 & 5 & A1 & 10.0807223 & -90.5 & 10.0806318 & 2 \\
\hline & & 9 & 7 & A1 & 10.0807223 & -90.5 & 10.0806318 & 2 \\
\hline & & 3 & 1 & A1 & 10.0785076 & -81.9 & 10.0784257 & 2 \\
\hline & & 5 & 3 & A1 & 10.0785076 & -81.9 & 10.0784257 & 2 \\
\hline & & 7 & 5 & $\mathrm{E} 2$ & 10.0560237 & 2.2 & 10.0560259 & 2 \\
\hline & & 9 & 7 & E2 & 10.0560237 & 2.2 & 10.0560259 & 2 \\
\hline & & 3 & 1 & E2 & 10.0538091 & -2.1 & 10.0538069 & 2 \\
\hline & & 5 & 3 & E2 & 10.0538091 & -2.1 & 10.0538069 & 2 \\
\hline & & 3 & 3 & E2 & 10.0627530 & -6.2 & 10.0627468 & 2 \\
\hline & & 5 & 5 & E2 & 10.0601976 & 6.8 & 10.0602043 & 2 \\
\hline & & 7 & 7 & E2 & 10.0470798 & 3.2 & 10.0470830 & 2 \\
\hline & & 7 & 5 & I1 & 9.9898437 & 48.4 & 9.9898921 & 2 \\
\hline & & 9 & 7 & I1 & 9.9899365 & 48.8 & 9.9899854 & 2 \\
\hline & & 3 & 1 & I1 & 9.9878817 & 51.5 & 9.9879332 & 2 \\
\hline & & 5 & 3 & I1 & 9.9877864 & 41.6 & 9.9878280 & 2 \\
\hline & & 5 & 5 & $\mathrm{I} 1$ & 9.9938456 & 53.2 & 9.9938988 & 2 \\
\hline & & 7 & 7 & I1 & 9.9813614 & 49.6 & 9.9814110 & 2 \\
\hline & & 7 & 5 & I2 & 9.9960612 & 138.8 & 9.9962000 & 2 \\
\hline & & 9 & 7 & $\mathrm{I} 2$ & 9.9961429 & 140.1 & 9.9962830 & 2 \\
\hline & & 3 & 1 & $\mathrm{I} 2$ & 9.9940807 & 140.2 & 9.9942210 & 2 \\
\hline & & 5 & 3 & $\mathrm{I} 2$ & 9.9939967 & 135.7 & 9.9941324 & 2 \\
\hline & & 5 & 5 & $\mathrm{I} 2$ & 10.0000616 & 144.5 & 10.0002061 & 2 \\
\hline
\end{tabular}


Table 7: continue: Torsion-rotation transition frequencies $(\mathrm{K}=0)$ of the main isotopologue $\left(\mathrm{CH}_{3}\right)_{3}{ }^{120} \mathrm{Sn}^{35} \mathrm{Cl}$.

\begin{tabular}{|c|c|c|c|c|c|c|c|c|}
\hline$J^{\prime}$ & $J$ & $2 F^{\prime}$ & $2 F$ & Sym & $v_{\text {calc }} / \mathrm{GHz}$ & $\delta v / \mathrm{kHz}$ & $v_{o b s} / \mathrm{GHz}$ & $U_{n c} / \mathrm{kHz}$ \\
\hline & & 7 & 7 & $\mathrm{I} 2$ & 9.9875710 & 258.3 & 9.9878293 & 2 \\
\hline & & 7 & 5 & I3 & 9.8515035 & -268.6 & 9.8512349 & 2 \\
\hline & & 9 & 7 & I3 & 9.8518284 & -265.0 & 9.8515634 & 2 \\
\hline & & 3 & 1 & I3 & 9.8500280 & -263.5 & 9.8497646 & 2 \\
\hline & & 5 & 3 & I3 & 9.8496994 & -269.5 & 9.8494300 & 2 \\
\hline & & 5 & 5 & I3 & 9.8553401 & -170.8 & 9.8551693 & 2 \\
\hline & & 7 & 7 & I3 & 9.8436074 & -217.3 & 9.8433901 & 3 \\
\hline & & 5 & 5 & $\mathrm{I} 4$ & 9.8972457 & 65.1 & 9.8973108 & 5 \\
\hline & & 7 & 7 & $\mathrm{I} 4$ & 9.8853366 & 69.4 & 9.8854060 & 5 \\
\hline & & 9 & 7 & $\mathrm{I} 4$ & 9.8936314 & 64.1 & 9.8936955 & 2 \\
\hline & & 7 & 5 & $\mathrm{I} 4$ & 9.8933746 & 61.4 & 9.8934360 & 2 \\
\hline & & 3 & 1 & I4 & 9.8917640 & 65.4 & 9.8918293 & 2 \\
\hline & & 5 & 3 & $\mathrm{I} 4$ & 9.8915037 & 59.9 & 9.8915635 & 2 \\
\hline \multirow[t]{13}{*}{4} & 3 & 11 & 9 & A1 & 13.4406431 & -139.4 & 13.4405037 & 2 \\
\hline & & 9 & 7 & A1 & 13.4406431 & -139.4 & 13.4405037 & 2 \\
\hline & & 7 & 7 & A1 & 13.4437792 & -135.3 & 13.4436439 & 2 \\
\hline & & 7 & 5 & A1 & 13.4396054 & -142.0 & 13.4394634 & 2 \\
\hline & & 5 & 3 & A1 & 13.4396054 & -142.0 & 13.4394634 & 2 \\
\hline & & 9 & 9 & A1 & 13.4316991 & -138.1 & 13.4315610 & 2 \\
\hline & & 5 & 5 & A1 & 13.4485493 & -144.4 & 13.4484049 & 2 \\
\hline & & 11 & 9 & $\mathrm{E} 2$ & 13.4077239 & -12.7 & 13.4077113 & 2 \\
\hline & & 9 & 7 & E2 & 13.4077239 & -12.7 & 13.4077113 & 2 \\
\hline & & 7 & 7 & E2 & 13.4108601 & -9.2 & 13.4108510 & 2 \\
\hline & & 7 & 5 & E2 & 13.4066863 & -14.2 & 13.4066721 & 2 \\
\hline & & 5 & 3 & E2 & 13.4066863 & -14.2 & 13.4066721 & 2 \\
\hline & & 9 & 9 & $\mathrm{E} 2$ & 13.3987800 & -12.3 & 13.3987677 & 2 \\
\hline
\end{tabular}


Table 7: continue: Torsion-rotation transition frequencies $(\mathrm{K}=0)$ of the main isotopologue $\left(\mathrm{CH}_{3}\right)_{3}{ }^{120} \mathrm{Sn}^{35} \mathrm{Cl}$.

\begin{tabular}{|c|c|c|c|c|c|c|c|}
\hline$J^{\prime} \quad J$ & $2 F^{\prime}$ & $2 F$ & Sym & $\nu_{\text {calc }} / \mathrm{GHz}$ & $\delta v / \mathrm{kHz}$ & $v_{o b s} / \mathrm{GHz}$ & Unc/kHz \\
\hline & 5 & 5 & E2 & 13.4156302 & -16.6 & 13.4156136 & 2 \\
\hline & 11 & 9 & I1 & 13.3332861 & 78.3 & 13.3333644 & 2 \\
\hline & 9 & 7 & I1 & 13.3332115 & 77.0 & 13.3332885 & 2 \\
\hline & 7 & 7 & I1 & 13.3362446 & 82.1 & 13.3363267 & 2 \\
\hline & 9 & 9 & I1 & 13.3246364 & 79.4 & 13.3247158 & 2 \\
\hline & 5 & 5 & I1 & 13.3408922 & 74.8 & 13.3409670 & 2 \\
\hline & 7 & 5 & I1 & 13.3322427 & 76.5 & 13.3323192 & 2 \\
\hline & 5 & 3 & I1 & 13.3323178 & 75.0 & 13.3323928 & 2 \\
\hline & 11 & 9 & $\mathrm{I} 2$ & 13.3397751 & 158.8 & 13.3399339 & 2 \\
\hline & 9 & 7 & $\mathrm{I} 2$ & 13.3397073 & 158.9 & 13.3398662 & 2 \\
\hline & 7 & 7 & $\mathrm{I} 2$ & 13.3427369 & 160.6 & 13.3428975 & 3 \\
\hline & 7 & 5 & $\mathrm{I} 2$ & 13.3387364 & 157.4 & 13.3388938 & 2 \\
\hline & 5 & 3 & $\mathrm{I} 2$ & 13.3388047 & 157.4 & 13.3389621 & 2 \\
\hline & 9 & 9 & $\mathrm{I} 2$ & 13.3311354 & 276.7 & 13.3314121 & 3 \\
\hline & 5 & 5 & $\mathrm{I} 2$ & 13.3473759 & 158.2 & 13.3475341 & 3 \\
\hline & 11 & 9 & I3 & 13.1876048 & -181.8 & 13.1874230 & 3 \\
\hline & 9 & 7 & I3 & 13.1874099 & -179.6 & 13.1872303 & 3 \\
\hline & 7 & 5 & I3 & 13.1865243 & -185.5 & 13.1863388 & 3 \\
\hline & 5 & 3 & I3 & 13.1867198 & -177.3 & 13.1865425 & 3 \\
\hline & 9 & 9 & I4 & 13.2225056 & 106.4 & 13.2226120 & 2 \\
\hline & 5 & 5 & $\mathrm{I} 4$ & 13.2383539 & 99.6 & 13.2384535 & 2 \\
\hline & 7 & 7 & $\mathrm{I} 4$ & 13.2337665 & 107.7 & 13.2338742 & 2 \\
\hline & 11 & 9 & I4 & 13.2309643 & 105.5 & 13.2310698 & 2 \\
\hline & 9 & 7 & I4 & 13.2308004 & 104.4 & 13.2309048 & 2 \\
\hline & 7 & 5 & $\mathrm{I} 4$ & 13.2298954 & 103.3 & 13.2299987 & 2 \\
\hline & 5 & 3 & $\mathrm{I} 4$ & 13.2300599 & 104.6 & 13.2301646 & 2 \\
\hline
\end{tabular}


Table 7: continue: Torsion-rotation transition frequencies $(K=0)$ of the main isotopologue $\left(\mathrm{CH}_{3}\right)_{3}{ }^{120} \mathrm{Sn}^{35} \mathrm{Cl}$.

\begin{tabular}{|c|c|c|c|c|c|c|c|c|}
\hline$J^{\prime}$ & $J$ & $2 F^{\prime}$ & $2 F$ & Sym & $\nu_{\text {calc }} / \mathrm{GHz}$ & $\delta v / \mathrm{kHz}$ & $v_{o b s} / \mathrm{GHz}$ & Unc/kHz \\
\hline \multirow[t]{26}{*}{5} & 4 & 13 & 11 & A1 & 16.8006155 & -197.7 & 16.8004178 & 2 \\
\hline & & 11 & 9 & A1 & 16.8006155 & -197.7 & 16.8004178 & 2 \\
\hline & & 9 & 7 & A1 & 16.8000020 & -199.2 & 16.7998028 & 2 \\
\hline & & 7 & 5 & A1 & 16.8000020 & -199.2 & 16.7998028 & 2 \\
\hline & & 9 & 7 & E2 & 16.7588727 & -13.8 & 16.7588589 & 2 \\
\hline & & 7 & 5 & E2 & 16.7588727 & -13.8 & 16.7588589 & 2 \\
\hline & & 11 & 9 & E2 & 16.7594863 & -19.4 & 16.7594669 & 2 \\
\hline & & 13 & 11 & E2 & 16.7594863 & -19.4 & 16.7594669 & 2 \\
\hline & & 7 & 7 & E2 & 16.7678167 & -20.1 & 16.7677966 & 2 \\
\hline & & 9 & 7 & $\mathrm{I} 1$ & 16.6801049 & 99.9 & 16.6802048 & 2 \\
\hline & & 7 & 5 & I1 & 16.6801619 & 100.2 & 16.6802621 & 2 \\
\hline & & 11 & 9 & I1 & 16.6806823 & 100.8 & 16.6807831 & 2 \\
\hline & & 13 & 11 & I1 & 16.6807391 & 101.7 & 16.6808408 & 2 \\
\hline & & 9 & 9 & I1 & 16.6831380 & 107.5 & 16.6832455 & 2 \\
\hline & & 11 & 11 & $\mathrm{I} 1$ & 16.6720326 & 68.2 & 16.6721008 & 2 \\
\hline & & 9 & 7 & $\mathrm{I} 2$ & 16.6865448 & 159.9 & 16.6867046 & 2 \\
\hline & & 7 & 5 & $\mathrm{I} 2$ & 16.6865978 & 160.5 & 16.6867583 & 2 \\
\hline & & 11 & 9 & $\mathrm{I} 2$ & 16.6871226 & 160.6 & 16.6872832 & 2 \\
\hline & & 13 & 11 & $\mathrm{I} 2$ & 16.6871755 & 161.1 & 16.6873366 & 2 \\
\hline & & 9 & 9 & $\mathrm{I} 2$ & 16.6895744 & 165.2 & 16.6897396 & 2 \\
\hline & & 9 & 7 & I3 & 16.5288961 & -126.4 & 16.5287697 & 2 \\
\hline & & 7 & 5 & I3 & 16.5290197 & -126.6 & 16.5288931 & 2 \\
\hline & & 11 & 9 & I3 & 16.5294386 & -126.8 & 16.5293118 & 2 \\
\hline & & 13 & 11 & I3 & 16.5295621 & -126.9 & 16.5294352 & 2 \\
\hline & & 9 & 9 & I3 & 16.5318471 & -126.4 & 16.5317207 & 2 \\
\hline & & 9 & 9 & $\mathrm{I} 4$ & 16.5765047 & 98.6 & 16.5766033 & 2 \\
\hline
\end{tabular}


Table 7: continue: Torsion-rotation transition frequencies $(K=0)$ of the main isotopologue $\left(\mathrm{CH}_{3}\right)_{3}{ }^{120} \mathrm{Sn}^{35} \mathrm{Cl}$.

\begin{tabular}{|c|c|c|c|c|c|c|c|c|}
\hline$J^{\prime}$ & $J$ & $2 F^{\prime}$ & $2 F$ & Sym & $v_{\text {calc }} / \mathrm{GHz}$ & $\delta v / \mathrm{kHz}$ & $v_{o b s} / \mathrm{GHz}$ & Unc $/ \mathrm{kHz}$ \\
\hline \multirow{26}{*}{6} & \multirow{26}{*}{5} & 9 & 7 & $\mathrm{I} 4$ & 16.5735386 & 93.6 & 16.5736322 & 2 \\
\hline & & 7 & 5 & $\mathrm{I} 4$ & 16.5736463 & 94.6 & 16.5737409 & 2 \\
\hline & & 11 & 9 & $\mathrm{I} 4$ & 16.5740885 & 94.4 & 16.5741829 & 2 \\
\hline & & 13 & 11 & $\mathrm{I} 4$ & 16.5741961 & 95.4 & 16.5742915 & 2 \\
\hline & & 15 & 13 & A1 & 20.1605966 & -273.8 & 20.1603229 & 2 \\
\hline & & 13 & 11 & A1 & 20.1605966 & -273.8 & 20.1603229 & 2 \\
\hline & & 13 & 13 & $\mathrm{~A} 1$ & 20.1516527 & -273.5 & 20.1513792 & 2 \\
\hline & & 11 & 9 & A1 & 20.1601880 & -274.6 & 20.1599134 & 2 \\
\hline & & 9 & 7 & A1 & 20.1601880 & -274.6 & 20.1599134 & 2 \\
\hline & & 15 & 13 & E2 & 20.1112703 & -28.7 & 20.1112416 & 2 \\
\hline & & 13 & 11 & E2 & 20.1112703 & -28.7 & 20.1112416 & 2 \\
\hline & & 11 & 9 & E2 & 20.1108616 & -26.8 & 20.1108348 & 2 \\
\hline & & 9 & 7 & E2 & 20.1108616 & -26.8 & 20.1108348 & 2 \\
\hline & & 15 & 13 & I1 & 20.0307677 & 115.7 & 20.0308834 & 2 \\
\hline & & 13 & 11 & I1 & 20.0307248 & 115.6 & 20.0308403 & 2 \\
\hline & & 11 & 11 & $\mathrm{I} 1$ & 20.0327929 & 117.8 & 20.0329107 & 3 \\
\hline & & 13 & 13 & I1 & 20.0220182 & 117.4 & 20.0221356 & 3 \\
\hline & & 11 & 9 & I1 & 20.0303371 & 114.8 & 20.0304520 & 2 \\
\hline & & 9 & 7 & I1 & 20.0303801 & 114.4 & 20.0304946 & 2 \\
\hline & & 15 & 13 & $\mathrm{I} 2$ & 20.0369612 & 149.2 & 20.0371104 & 2 \\
\hline & & 13 & 11 & $\mathrm{I} 2$ & 20.0369205 & 149.6 & 20.0370701 & 2 \\
\hline & & 11 & 11 & $\mathrm{I} 2$ & 20.0389847 & 150.8 & 20.0391355 & 3 \\
\hline & & 13 & 13 & I2 & 20.0282279 & 152.0 & 20.0283799 & 3 \\
\hline & & 11 & 9 & I2 & 20.0365329 & 148.0 & 20.0366810 & 2 \\
\hline & & 9 & 7 & I2 & 20.0365737 & 147.7 & 20.0367214 & 2 \\
\hline & & 15 & 13 & I3 & 19.8746928 & -86.3 & 19.8746065 & 2 \\
\hline
\end{tabular}


Table 7: continue: Torsion-rotation transition frequencies $(K=0)$ of the main isotopologue $\left(\mathrm{CH}_{3}\right)_{3}{ }^{120} \mathrm{Sn}^{35} \mathrm{Cl}$.

\begin{tabular}{|c|c|c|c|c|c|c|c|}
\hline$J^{\prime} \quad J$ & $2 F^{\prime}$ & $2 F$ & Sym & $\nu_{\text {calc }} / \mathrm{GHz}$ & $\delta v / \mathrm{kHz}$ & $v_{o b s} / \mathrm{GHz}$ & Unc $/ \mathrm{kHz}$ \\
\hline & 13 & 11 & I3 & 19.8746100 & -87.2 & 19.8745228 & 2 \\
\hline & 11 & 11 & I3 & 19.8766480 & -83.7 & 19.8765643 & 3 \\
\hline & 13 & 13 & I3 & 19.8660706 & -79.7 & 19.8659909 & 3 \\
\hline & 11 & 9 & I3 & 19.8742394 & -85.9 & 19.8741535 & 2 \\
\hline & 9 & 7 & I3 & 19.8743223 & -87.0 & 19.8742353 & 2 \\
\hline & 11 & 11 & I4 & 19.9224777 & 81.2 & 19.9225589 & 3 \\
\hline & 13 & 13 & $\mathrm{I} 4$ & 19.9118692 & 43.0 & 19.9119122 & 2 \\
\hline & 15 & 13 & $\mathrm{I} 4$ & 19.9205092 & 36.3 & 19.9205455 & 2 \\
\hline & 13 & 11 & $\mathrm{I} 4$ & 19.9204355 & 36.0 & 19.9204715 & 2 \\
\hline & 11 & 9 & $\mathrm{I} 4$ & 19.9200615 & 35.5 & 19.9200970 & 2 \\
\hline & 9 & 7 & I4 & 19.9201353 & 35.6 & 19.9201709 & 2 \\
\hline
\end{tabular}

Table 8 summarizes the molecular constants obtained from a least-square fit of the $K=0$ torsion-rotation transitions of the main isotopologue $\left(\mathrm{CH}_{3}\right)_{3}{ }^{120} \mathrm{Sn}^{35} \mathrm{Cl}$ using XIAM (182 lines). As can be seen, molecular parameters such as the $B$ constant and quadrupole coupling constant $e Q q$ obtained from the XIAM fit are quite similar to the effective data obtained from the rigid rotor fits summarized in Table 4 . The obtained internal rotation barrier is $V_{3}=0.42372(16) \mathrm{kcal}\left(148.299(54) \mathrm{cm}^{-1}\right)$ very close to the theoretically predicted value of $150 \mathrm{~cm}^{-1}$. In addition, the angle determined between the $\mathrm{Sn}-\mathrm{Cl}$ bond and the internal rotor symmetry axis $\beta_{C}$ is $104.73(18)^{\circ}$, which is, again, very similar to the ab initio value (BP86/def-TZVPP/ecp-46-mwb(Sn)) of $105.1^{\circ}$ (Figure 2).

Based on the effective $B$-constants obtained for different isotopologues from our rigid rotor fits (Table 4) we determined the $\mathrm{Sn}-\mathrm{Cl}$ bond length to be $\mathrm{r}(\mathrm{Sn}$ $\mathrm{Cl})=2.35191(94) \AA$. The ab initio-value is $2.399 \AA$ (Figure 2). Furthermore, the experimentally determined $A$-constant of $2404.56(68) \mathrm{MHz}$ (Table 8 ) is fairly close to the calculated value of $A_{\text {calc }}=2330.67 \mathrm{MHz}$. These results are a good indication that density functional calculations including electron core potentials for a heavy element such as tin give quite reliable structural results. In this context it is interesting to draw a comparison to the related molecule $\left(\mathrm{CH}_{3}\right)_{3} \mathrm{SiCl}$. Both the $A$ and the $B$ constant are larger $(A=3051$.(68) $\mathrm{MHz}$ and 
Table 8

Spectroscopic constants of $\left(\mathrm{CH}_{3}\right)_{3}{ }^{120} \mathrm{Sn}^{35} \mathrm{Cl}$ derived from a least-square fit of the $K=0$ torsion-rotation transitions using XIAM (rotational constants $\mathrm{A}, \mathrm{B}$, and $\mathrm{C}$, centrifugal distortion constants $\mathrm{D}_{J}$, molecular quadrupole coupling constants eQq and barrier to internal rotation $\mathrm{V}_{3}$ ). $F_{0}$ is the rotational constant of the methyl group corresponding to $I_{\alpha}=3.199 \mathrm{u} \cdot \AA^{2}$ and has been kept fixed during the fitting procedure, $\mathrm{N}$ is the number of lines included in the fit, and $\sigma$ gives the standard deviation of the fit. $\theta$ describes the angle between the internal rotor axis and the inertial a axis, which is oriented along the $\mathrm{Sn}-\mathrm{Cl}$ bond. The numbers in parentheses give uncertainties in units of the last digit (type $\mathrm{A}, k=1$ [24].

\begin{tabular}{cccc}
\hline \hline quantity & value & quantity & value \\
\hline $\mathrm{A} / \mathrm{MHz}$ & $2404.56(68)$ & $\mathrm{V}_{3} / \mathrm{kcal}$ & $0.42372(16)$ \\
$\mathrm{B} / \mathrm{MHz}$ & $1677.3039(40)$ & $F_{0} / \mathrm{GHz}$ & 158.0 \\
$\mathrm{D}_{J} / \mathrm{kHz}$ & $0.133(66)$ & $\theta /{ }^{\circ}$ & $104.73(18)$ \\
$\mathrm{eQq} / \mathrm{MHz}$ & $-35.776(81)$ & $\mathrm{N}$ & 182 \\
& & $\sigma / \mathrm{kHz}$ & 129.5 \\
\hline \hline
\end{tabular}

Table 9

$V_{3}$-barrier heights for $\left(\mathrm{CH}_{3}\right)_{3} \mathrm{XCl}$-species $(\mathrm{X}=\mathrm{C}, \mathrm{Si}, \mathrm{Ge}, \mathrm{Sn})$, as determined from microwave studies.

\begin{tabular}{ll}
\hline \hline$X$ & $V_{3} / \mathrm{cm}^{-1}$ \\
\hline $\mathrm{C}$ & close to $\infty[6]$ \\
$\mathrm{Si}$ & $577[1]$ \\
$\mathrm{Ge}$ & $346.8(41)[3]$ \\
$\mathrm{Sn}$ & $148.299(54)$ (this work) \\
\hline \hline
\end{tabular}

$B=2197.382227(66) \mathrm{MHz}[1]$, respectively) than for $\left(\mathrm{CH}_{3}\right)_{3} \mathrm{SnCl}$, but with a similar $A-B$ of $853.6 \mathrm{MHz}$.

The different barrier heights for $\left(\mathrm{CH}_{3}\right)_{3} \mathrm{XCl}$-species $(\mathrm{X}=\mathrm{C}, \mathrm{Si}, \mathrm{Ge}, \mathrm{Sn})$ are summarized in Table 9. For the carbon species no internal rotation splittings have been resolved, so that $V_{3}$ can be assumed to be large. As expected, the barrier height decreases significantly from Si to Ge to Sn. Because of the large $\mathrm{X}-\mathrm{C}$ bond length in $\left(\mathrm{CH}_{3}\right)_{3} \mathrm{SnCl}$, it can be assumed that the origin of the remaining barrier height of $V_{3}=148.299(54) \mathrm{cm}^{-1}$ is mainly due to the $\mathrm{Sn}-\mathrm{C}$ bonding character, rather than due to steric effects. The resulting low barrier implies that, to correctly describe the internal dynamics and therefore the top-top communication for $\left(\mathrm{CH}_{3}\right)_{3} \mathrm{SnCl}$, multidimensional tunneling pathways, such as the concerted motion of three methyl groups at a time, also have to be taken into account (Section 4.4), in contrast to $\left(\mathrm{CH}_{3}\right)_{3} \mathrm{SiCl}$ and $\left(\mathrm{CH}_{3}\right)_{3} \mathrm{GeCl}$. 


\section{Conclusion}

The rotational spectra of several isotopologues of $\left(\mathrm{CH}_{3}\right)_{3} \mathrm{SnCl}$ have been recorded using pulsed molecular beam high-resolution microwave spectroscopy. One rotational $J+1 \leftarrow J$-transition was found to be split into several subgroups due to internal rotation motion of the three chemically and grouptheoretically equivalent methyl groups, as well as chlorine quadrupole coupling and numerous chlorine and tin isotopes.

PI group-theoretical approaches using the group $G_{162}$ have been developed and used to support an assignment of the complex torsion-rotation substructure, which consists of six $K=0$ and $11 K=1$ torsion-rotation states. We were able to theoretically devise a scheme to differentiate between torsional transitions by the determination of their Stark behavior. $\Delta K= \pm 1$-mixing effects between $K=0$ - and $K=1$-levels seem to be the reason for two unusual and unexpected observations for the $I_{i}$-symmetry species $(i=1-4)$ : linear Stark behavior for $I_{1^{-}}, I_{2}$ - and $I_{3}$-symmetry $K=0$ torsion-rotation transitions and additional $K=0$ quadrupole splittings for $I_{1}, I_{2}, I_{3}$ and $I_{4}$. The $K=1$ contributions (coefficients squared) to the $K=0$-levels are estimated to be about $1 \%$. As a result of a global fit including $182 \mathrm{~K}=0$ torsion-rotation transitions we were able to determine the barrier height for $\mathrm{CH}_{3}$ internal rotation to be $148.299(54) \mathrm{cm}^{-1}$. This is significantly lower than for the related species $\left(\mathrm{CH}_{3}\right)_{3} \mathrm{SiCl}$ and $\left(\mathrm{CH}_{3}\right)_{3} \mathrm{GeCl}$ and can be assumed to be mainly due to hindering from the chemical $\mathrm{Sn}-\mathrm{C}$ bond. As a next step we want to perform a quantitative study on the $\Delta K= \pm 1$-mixing.

In a subsequent study we want to investigate further the interaction between internal rotation and nuclear quadrupole coupling. Good candidates would be the series $\left(\mathrm{CH}_{3}\right)_{3} \mathrm{GeY}$ and $\left(\mathrm{CH}_{3}\right)_{3} \mathrm{SnY}$, with $\mathrm{Y}=\mathrm{Br}$ and I, since both bromine and iodine exhibit significantly stronger quadrupole coupling, so that we hope to find additional effects due to coupling between quadrupole and internal rotation.

\section{Acknowledgements}

Financial support of the Land Niedersachsen and the Deutsche Forschungsgemeinschaft is gratefully acknowledged. M.S. is grateful for a Postdoktorandenstipendium of the Deutsche Akademische Austauschdienst and the Akademiestipendium 2005 of the Berlin-Brandenburgische Akademie der Wissenschaften. 


\section{References}

[1] I. Merke, W. Stahl, S. Kassi, D. Petitprez, G. Wlodarczak, Internal rotation, quadrupole coupling, and structure of (ch3)3sicl studied by microwave spectroscopy and ab initio calculation, J. Mol. Spec. 216 (2002) 437-446.

[2] K. Voges, J. Gripp, H. Hartwig, H. Dreizler, Analysis of torsion in a threetop molecule. torsional barrier and moment of inertia of trimethyl ethynyl germane., Z. Naturforsch. 51a (1996) 299-305.

[3] M. Schnell, J.-U. Grabow, Internal dynamics in organometallic molecules: Rotational spectrum of $\left(\mathrm{ch}_{3}\right)_{3}$ gecl, Phys. Chem. Chem. Phys. 8 (2006) 22252231.

[4] M. Schnell, J.-U. Grabow, Multi-dimensional large-amplitude motions: Toptop communication through chemical bonds, Angew. Chem. Int. Ed. 45 (2006) 3465-3470, and Supporting Information.

[5] I. Merke, A. Lüchow, W. Stahl, Internal rotation, quadrupole coupling and structure of (ch3)3sii studied by microwave spectroscopy and ab initio calculations, J. Mol. Struct. 780-781 (2006) 295-299.

[6] M. C. Ellis, A. C. Legon, C. A. Rego, D. J. Millen, J. Mol. Struct. 200 (1989) 353-359.

[7] J. R. Durig, K. L. Hellams, Spectra and structure of organogermanes xviii. microwave spectrum of trimethylchlorogermane, J. Mol. Struct. 29 (1975) 349356.

[8] J.-U. Grabow, W. Stahl, H. Dreizler, A multioctave coaxially oriented beamresonator arrangement fourier-transform microwave spectrometer, Rev. Sci. Instrum. 67 (1996) 4072-4084.

[9] M. Schnell, D. Banser, J.-U. Grabow, Coaxially aligned electrodes for starkeffect applied in resonators using a supersonic jet fourier transform microwave spectrometer, Rev. Sci. Instrum. 75 (2004) 2111-2115.

[10] J. M. L. J. Reinartz, A. Dymanus, Chem. Phys. Lett. 24 (1974) 346-351.

[11] C. H. Townes, A. L. Schawlow, Microwave Spectroscopy, Dover Publications, New York, 1975.

[12] K. D. Möller, H. G. Andresen, On the theory of torsional vibrations of (ch3)3-x type molecules, J. Chem. Phys. 39 (1963) 17-22.

[13] K. K. Lehmann, B. H. Pate, G162: Molecular symmetry group of t-butane and other three equivalent methyl molecules, J. Mol. Spec. 144 (1990) 443-445.

[14] P. Groner, Nonrigid symmetry groups of molecular trimers and 3-rotor molecules, Spectrochim. Acta 49A (1993) 1935-1946.

[15] G. Herzberg, Infrared and Raman Spectra of Polyatomic Molecules, Van Nostrand, Princeton, NJ, 1945. 
[16] G. Herzberg, Electronic Spectra and Electronic Structure of Polyatomic Molecules, Van Nostrand, Princeton, NJ, 1966.

[17] J. T. Hougen, B. M. DeKoven, T, J. Mol. Spec. 98 (1983) 375-391.

[18] J. K. G. Watson, J. Mol. Spec. 50 (1974) 281-285.

[19] M. Hamermesh, Group Theory, Addison-Wesley, Reading,MA, 1962.

[20] N. Ohashi, J. T. Hougen, Application of permutation-inversion group theory to the interpretation of the microwave absorption spectrum of dimethyl methylphosphonate, J. Mol. Spec. 211 (2002) 119-126.

[21] J. T. Hougen, A rotational hamiltonian for the ground vibrational-state of hydrazine, J. Mol. Spec. 89 (1981) 296-327.

[22] Z. Kisiel, B. A. Pietrewicz, P. W. Fowler, A. C. Legon, E. Steiner, J. Phys. Chem. A 104 (2000) 6970-6978.

[23] B. Kleiböhmer, W. Kasten, J. Gripp, SYM2QS, Christian-Albrechts-Universität, Kiel, 1992.

[24] B. E. Taylor, C. E. Kuyatt, Guidelines for evaluating and expressing the uncertainty of nist measurement results, NIST Technical Note 1297, available from http://physics.nist.gov/cuu/Uncertainty/index.html.

[25] H. Hartwig, H. Dreizler, The microwave spectrum of trans-2,3-dimethyloxirane in torsional excited states, Z. Naturforsch. 51a (1996) 923-932.

[26] J. Rottstegge, H. Hartwig, H. Dreizler, J. Mol. Struct. 478 (1999) 37-47. 